\title{
AgGREGATION-INDUCED EMISSION: A CHALLENGE FOR COMPUTATIONAL CHEMISTRY. THE EXAMPLE OF TPA-BMO.
}

\author{
Laure de Thieulloy \\ Chimie ParisTech \\ PSL Research University, CNRS \\ Institute of Chemistry for Life \\ and Health Sciences (i-CLeHS) \\ F-75005 Paris, France \\ Laura Le Bras* \\ Chimie ParisTech \\ PSL Research University, CNRS \\ Institute of Chemistry for Life \\ and Health Sciences (i-CLeHS) \\ F-75005 Paris, France \\ laura.lebras@gmail.com

\section{Benoît Zumer} \\ Chimie ParisTech \\ PSL Research University, CNRS \\ Institute of Chemistry for Life \\ and Health Sciences (i-CLeHS) \\ F-75005 Paris, France \\ Claire Lemarchand \\ CEA/DAM/DIF \\ 91297 Arpajon Cedex, France \\ Université Paris-Saclay, CEA \\ Laboratoire Matière sous Conditions Extrêmes \\ 91680, Bruyères-le-Chatel, France.

\section{Carlo Adamo} \\ Chimie ParisTech - PSL Research \\ University, CNRS, Institute of Chemistry for Life \\ and Health Sciences (i-CLeHS) \\ F-75005 Paris, France \\ Institut Universitaire de France \\ 103 Bd Saint-Michel, F-75005 Paris - France \\ Nicolas Pineau \\ CEA/DAM/DIF \\ 91297 Arpajon Cedex, France \\ Université Paris-Saclay, CEA \\ Laboratoire Matière sous Conditions Extrêmes \\ 91680, Bruyères-le-Chatel, France.

\section{Aurélie Perrier*} \\ Chimie ParisTech - PSL Research \\ University, CNRS, Institute of Chemistry for Life \\ and Health Sciences (i-CLeHS) \\ F-75005 Paris, France \\ Université de Paris, F-75006 Paris, France \\ aurelie.perrier-pineau@u-paris.fr
}

March 24, 2021 


\begin{abstract}
A multi-scale and multi-environment computational approach is proposed to study of the modulation of the emission behavior of the triphenylamine (Z)-4-benzylidene-2methyloxazol-5(4H)-one (TPA-BMO) molecule [Tang et al., J. Phys. Chem. C 119, 21875 (2015)]. Going from (TD-)DFT calculations to classical Molecular Dynamics simulations through the hybrid ONIOM QM/QM' approach and the in situ chemical polymerization methodology, we have rationalized distinct photophysical phenomena: (1) in low-polar solvents, a polarity-dependent solvatochromic effect as well as a modulation of the emission quantum yield, attributed to possible photophysical energy dissipation caused by lowfrequency vibrational modes, (2) in the aggregate, a subtle competition between an excitonic coupling and a restriction of the intramolecular vibrations leading to an Aggregation-Induced Emission behavior, and (3) in the polymer matrix, an antagonist effect between the loss of global flexibility and the presence of vibrational modes similar to those observed in solution, explaining a similar emissive behavior within the polymer.
\end{abstract}

\title{
1 Introduction
}

Aggregate-Caused Quenching (ACQ), Aggregate-Induced Emission (AIE) and Crystallized-Induced Emission (CIE) can impact the emission properties of fluorophores. The first phenomenon, known since 1954 thanks to the work of Förster, [1, 2] is characteristic of plane and aromatic organic molecules. When aggregated, these molecules exhibit intermolecular interactions due to $\pi$-stacking, and the subsequent electronic couplings decrease the fluorescence quantum yield $\left(\Phi_{F}\right)$. The second phenomenon, was revealed in 2001 by Tang and coworkers with the highly flexible hexaphenlysilole molecule [3]. Once aggregated, the motions of the phenyl peripheral groups are no longer possible: the non-radiative deexcitation path due to rotational and /or vibrational deexcitation, observed in solution, is thus impeded and this restriction favors the radiative deexcitation channel [4, 5, 6]. This mechanism, known as "Intramolecular Vibration / Rotation Restrictions" (RIV / RIR), is also possible in the crystalline phase, leading to the so-called CIE phenomenon. In addition to RIV and RIR, theoretical studies have shown the existence of other mechanisms that may explain AIE and CIE such as the RACI model (Restricted Access to a Conical Intersection). Indeed, the investigation of the potential energy surfaces (PES) can reveal the existence of a conical intersection between the ground state (GS) and the first excited state (ES) which is accessible in solution and responsible for a rapid non-radiative decay. This CI can be energetically destabilized in condensed phases, thus impeding the non-radiative deactivation pathway and favoring the emission [7]. 
(a) TPA-(Z)-BMO

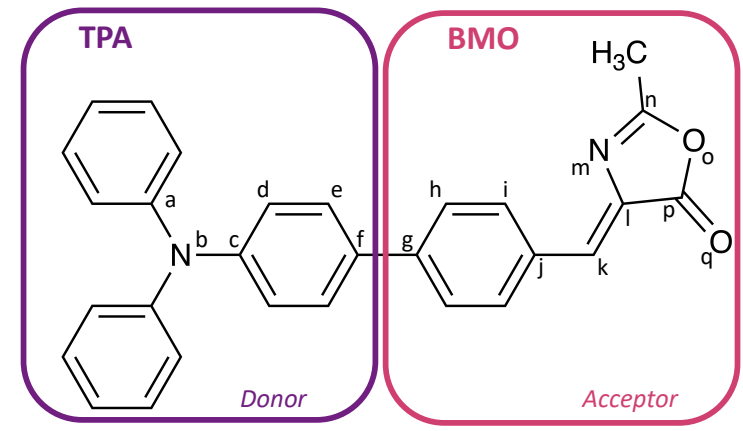

(b) TPA-(E)-BMO

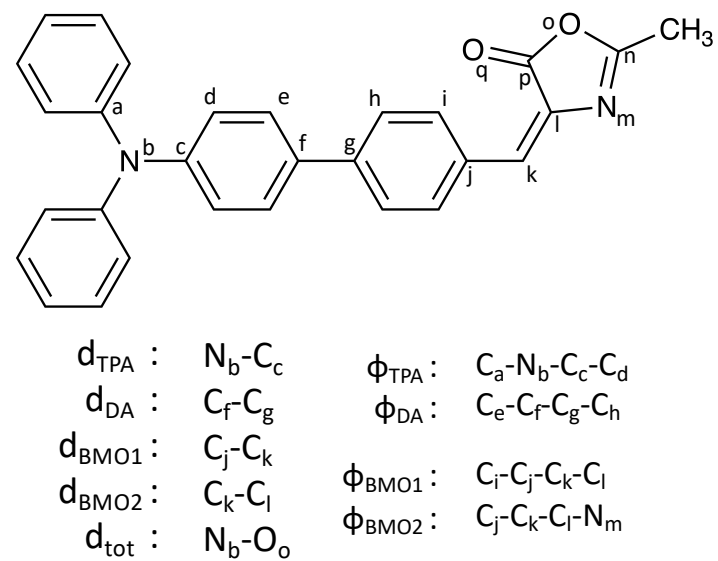

Figure 1: (a) Representation of the $(\mathbf{Z})$ isomer and (b) (E) isomer of the TPA-BMO molecule. The atom numbering is provided along with the definition of selected distances and dihedral angles.

In this work, we are interested in the Triphenylamine (Z)-4-benzylidene-2-methyloxazol-5(4H)-one (TPABMO) molecule (Fig. 1) which is a molecule synthesized via the condensation of an aldehyde and an $\mathrm{N}$-acetylglycine [8]. The acceptor group (BMO) is placed in para position of the TPA donor group (D) allowing an electronic delocalization over the whole molecular structure.

The interest for this molecule lies in the modulation of its fluorescence properties depending on the environment considered. It can be seen in Table 1 that in solution, for low-polar solvents, when the polarity of the solvent increases, the fluorescence quantum yield decreases while the emission wavelength, $\lambda_{\text {em }}$ is red-shifted [8]. In a strongly polar solvent such as acetonitrile (ACN), a dual fluorescence is observed. In aggregate, there is an enhancement of the radiative decay resulting in an AIE effect. More precisely, there is an increase of the photoluminescence (PL) intensity when the water fraction $\left(f_{w}\right)$ in DMSO-water mixtures increases. The PL intensity reaches a maximum for $f_{w}=70 \%$ with $\lambda_{e m}=573 \mathrm{~nm}$ [9]. In the solid phase, TPA-BMO emits in the red region $\left(\lambda_{e m}=635 \mathrm{~nm}\right)$ with a decrease of the $\Phi_{F}$ value compared to non-polar solvents [9, 10]. Finally, within a polymer matrix, the position of the emission band shifts towards longer wavelengths as the polarity of 
Table 1: Experimental optical properties of TPA-BMO in different environments: dilute solution (low polar solvents), aggregate, crystal and within a polymer film. $\lambda_{\max }$ is the maximum absorption wavelength, $\lambda_{e m}$ is the emission wavelength and $\Phi_{F}$ the fluorescence quantum yield (in \%). TLN stands for toluene, DXN $=$ Dioxane, $\mathrm{THF}=$ Tetrahydrofuran, $\mathrm{PB}=$ Polybutadiene, $\mathrm{PMMA}=$ Polymethyl methacrylate and $\mathrm{PEG}=$ Polyethylene glycol.

\begin{tabular}{|c|c|c|c|c|c|c|c|c|}
\hline & \multicolumn{3}{|c|}{ Solution[8] } & \multirow[t]{2}{*}{ Aggregate 9} & \multirow[t]{2}{*}{ Crystal } & \multicolumn{3}{|c|}{ Polymer[11] } \\
\hline & TLN & DXN & THF & & & PB & PMMA & PEG \\
\hline$\overline{\lambda_{\max }}$ & 419 & 406 & 407 & 425 & & & & \\
\hline$\lambda_{e m}$ & 509 & 530 & 576 & 573 & 597 & 499 & 525 & 567 \\
\hline$\Phi_{F}$ & 0.68 & 0.62 & 0.51 & & 0.19 & & & \\
\hline
\end{tabular}

the polymer chains increases [11]. The relative PL intensity is not discussed in this experimental work but there is no emission quenching when TPA-BMO is embedded in a polymer film.

Therefore, the TPA-BMO fluorophore presents a large modification of its emission properties while changing the environment. This AIEgen (i.e. chromophore exhibiting AIE) is a potential candidate for the development of luminescent solar concentrators (LSC) [12, 13]. In these devices, the AIEgen is embedded in a polymer matrix and controlling the AIE phenomenon in this environment represents a real challenge. In this context, theoretical studies represent a valuable tool to rationalize the emission properties of the fluorophore in these different environments [14, 15, 16, 17, 18]. In this work, we will be interested in three different environments: solution (low polar solvents), aggregate and polymer matrix. Our purpose is to "play" with the different methods relying on computational chemistry and molecular modeling to set up tailored calculation schemes able to reproduce and rationalize the experimental observations in these three environments. Due to the diversity and complexity of these different environments, we propose a qualitative analysis aimed at highlighting the photophysical phenomena leading to emission modulation. To this purpose, we will rely on previous models that we have proposed to model the AIE effect with the help of Density Functional Theory (DFT) and its Time-Dependent counterpart (TD-DFT), as well as Molecular Dynamics (MD) and hybrid QM/QM' calculations [14, 15, 16]. To investigate the behaviour of TPA-BMO in a polymer matrix, the model that we have recently developed to propose a realistic arrangement of a chromophore embedded in a polymer film will be considered [19].

After a presentation of the computational strategies, the absorption and emission properties of TPA-BMO in low-polar solvents, within an aggregate and embedded in a polymer matrix will be successively discussed.

\section{Computational details}

For the three environments, geometry optimizations, frequency calculations as well as the calculations of the absorption and emission properties, electronic energy transfer (EET) and Huang-Rhys (HR) factors are performed with the Gaussian 16 package [20]. 


\subsection{Solvated systems}

In solution, the geometries of the ground state $S_{0}$ are optimized using the DFT formalism while the study of the excited states relies on the TD-DFT formalism. In the course of the geometry optimization, we ensure that we obtain true minima on the potential energy surface (PES) by calculating the vibrational frequencies.

For both the geometry optimization and the calculation of the optical properties, we chose the exchangecorrelation functional (XCF) through a benchmark study performed with four different XCF: two global hybrids (PBE0 [21, 22] and B3LYP [23]), one range separated hybrid (RSH) (CAM-B3LYP) [24] and a meta-GGA functional (M06-2X)[25]. Each of this XCF has been associated with the 6-311G+(d,p) basis set. From this benchmark study detailed in the Supporting Information, we have selected the M06-2X//M06-2X calculation scheme which provides a calculated vertical excitation wavelength $\lambda_{a b s}$ equal to $377 \mathrm{~nm}$ in hexane. $\lambda_{a b s}$ corresponds to the excitation wavelength with the highest $f$ value in the low-lying excitation energy region. This leads to a satisfactory $\Delta \mathrm{E}_{\text {calc-exp }}$ deviation between calculated and experimental excitation energies of $0.25 \mathrm{eV}$.

The solvent is implicitly modeled via the Integral Equation Formalism Polarizable Continuum Model (IEFPCM) [26]. To check the validity of this approach regarding the computation of the absorption and emission properties, we have considered different solvents of increasing polarity: hexane (HXN), 1,4-dioxane (DXN), toluene (TLN), tetrahydrofuran (THF), dimethyl sulfoxide (DMSO), acetone (ACT) and acetonitrile (ACN). The ground state (GS) and excited state (ES) geometry optimizations as well as the prediction of the absorption and emission properties are performed in the equilibrium limit using the linear-response (LR) PCM scheme. The ground $\rho^{G S}(\mathrm{r})$ and excited state $\rho^{E S}(\mathrm{r})$ densities are computed with the help of the cubegen utility provided by the Gaussian16 suite and all density derived indexes were computed using the DctViaCube software [27, 28]. This index allows to define the spatial extent $D_{C T}$ corresponding to a given transition, using only the $\rho^{G S}(\mathrm{r})$ and $\rho^{E S}(\mathrm{r})$ density distributions. The definition of $D_{C T}$ (expressed in $\AA$ ) as well as the amount of charge transfer $q_{C T}$ (expressed as a function of the elementary charge e), is recalled in the Supporting Information section.

The fluorescence quantum yield, $\Phi_{F}$, is evaluated qualitatively by calculating the so-called Huang-Rhys (HR) factors. These dimensionless electron-vibration coupling constants allow, for an emission process, to represent the variation of the vibrational mode $j$ during the deexcitation process, $\mathrm{S}_{j} \rightarrow \mathrm{S}_{0}$ :

$$
H R_{j}=\frac{\omega_{j} D_{j}^{2}}{2 \hbar}
$$


where $\omega_{j}$ and $D_{j}$ are the frequency of the vibrational mode $j\left(\mathrm{~cm}^{-1}\right)$ and the displacement vector of this mode between the equilibrium positions of the $S_{j}$ and $S_{0}$ states [29, 14, 15]. These factors lead to the total reorganisation energy $E_{\text {reorg }}$ which is expressed as the sum over all the vibrational modes, $j$, of the product of the HR factor and the corresponding frequency:

$$
E_{\text {reorg }}=\sum_{j} H R_{j} \cdot \omega_{j}
$$

Some MD simulations have been carried out with the AMBER18 package[30] in a water solvent box. The simulation box corresponds to one TPA-BMO molecule surrounded by 4348 water molecules within a 20 A cubic box. The General Amber Force Field (GAFF)[31] is used to describe TPA-BMO and the water molecules are described with the TIP3P model [32]. For TPA-BMO, the atomic charges are obtained from the parametrization procedure applied in GAFF, that is to say using HF/6-31G(d) RESP charges. To validate the GAFF force field, we have compared the structures minimized in vacuum (i) with GAFF within the AMBER18 package and (ii) at the M06-2X/6-311g+(d,p) level with Gaussian16. The comparison of the structural parameters obtained with these two approaches is discussed in Supporting Information and shows that GAFF is able to provide accurate structural information for TPA-BMO.

The particle mesh Ewald (PME) method [33, 34] is used to model the electrostatic interactions and a cut-off of $8 \AA$ is considered. After an energy minimization using the steepest descent algorithm and a heating of the system from 0 to $300 \mathrm{~K}$ during $100 \mathrm{ps}$ in the NVT ensemble, an equilibration simulation is first performed in the NPT ensemble for $2 \mathrm{~ns}$ with a $1 \mathrm{fs}$ time step. The reference pressure (1 bar) and the temperature ( $300 \mathrm{~K})$ are incorporated with the Berendsen method [35]. The subsequent MD simulations are carried out in the NVT ensemble for $10 \mathrm{~ns}$. The results of the MD simulations are then analysed with CPPTRAJ software [36] within the AMBER18 package.

\subsection{Aggregate phase}

To model the aggregate phase, MD simulations are also performed with the AMBER18 suite. The initial configuration of the aggregate is generated by the PACKMOL package [37]: 30 TPA-BMO molecules are placed in a $30 \AA$ side cubic box. This box is nested in a $80 \AA$ side cubic box containing 17124 water molecules, this simulation box is represented in Fig. 2 a). The TPA-BMO molecules are more concentrated at the center of the simulation box to avoid a time-consuming aggregation process starting from a uniform distribution [38]. Then, the same protocol as the one described for one TPA-BMO molecule in solution is applied. At the end of the NPT equilibrium simulation, we have checked that the system is at equilibrium and found a small 
(a)

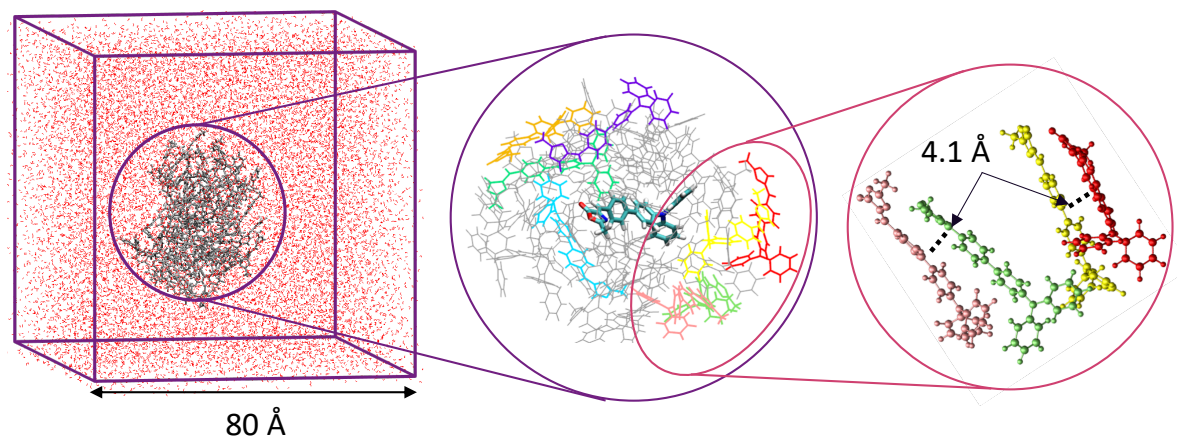

Figure 2: (a) Representation of the TPA-BMO aggregate in the simulation water box with an enlarged view of (b) the aggregate with an highlighted molecule at the center and (c) the dimer organization for molecules located at the periphery of the aggregate.

contraction of the cubic box (the edge length was reduced by $0.55 \%$ ). The subsequent MD simulations are carried out in the NVT ensemble for $10 \mathrm{~ns}$.

We have randomly selected 10 TPA-BMO molecules within the aggregate and regularly extracted snapshots along the $10 \mathrm{~ns}$ trajectory. The structural parameters of these 10 molecules are analyzed along the trajectory. Concerning the optical properties, we have selected some molecules at the center of the aggregate (Fig. 2 (b)) and at the edge (Fig. 2(c)). To calculate the absorption properties, we have relied on the ONIOM model and more precisely, on a QM/QM' strategy with polarisation embedding [39]. The QM method is M06-2X/6-311+G(d,p) while the QM' method corresponds to HF/3-21G. For a TPA-BMO molecule localized at the center of the aggregate, the model (or "high level", HL) region corresponds to the molecule of interest while the other TPA-BMO molecules (29 molecules) are described at the QM' level. To describe the absorption properties of a molecule at the periphery of the aggregate, several computational methodologies are compared in the Supporting Information section (Table S4, Fig. S4 and S5). The strategy adopted is as follows (Fig. S4): (i) the $\mathrm{HL}$ region corresponds to the molecule of interest and to the $\mathrm{H}_{2} \mathrm{O}$ molecules present in the first solvation shell (that typically encompasses 1 to 4 molecules), (ii) the 10 TPA-BMO molecules closest to the molecule of interest are described at the low QM' level, the other are removed (iii) the ONIOM-PCM model is considered [40, 41].

To calculate the emission properties, while optimizing the geometry of the $S_{1}$ state, the molecules included in the model system have the possibility to relax, while those constituting the surrounding system are kept frozen. 


\subsection{Polymer}

The TPA-BMO/polymer simulations are carried out with STAMP, a massively parallelised MD software developed at CEA/DAM/DIF [42, 43, 44, 45] following the procedure recently developed to describe the properties of a chromophore embedded in a polymer matrix [19]. We have considered a model cis-1,4polybutadiene matrix (referred to as polybutadiene or PB in the following).

We have used GAFF for TPA-BMO and the all-atom Optimized Potentials for Liquid Simulations force field (OPLS-AA) [46] for the polybutadiene chains as done in previous works for this polymer [45]. The van der Waals and electrostatic cross-interactions between TPA-BMO and polybutadiene are defined by a 12-6 Lennard-Jones (LJ) potential, with the $\varepsilon$ and $\sigma$ parameters obtained by the usual geometric mixing rules between the pure GAFF and OPLS-AA non-bonded parameters. The long-range contribution of the Coulomb interactions is computed with the Reaction Field method, using a cutoff radius of $10 \AA$ and a relative permittivity $\varepsilon / \varepsilon_{0}=2$, corresponding to the experimental value of polybutadiene [47, 48, 49].

We have used the so-called controlled-like polymerization algorithm, noted CLP hereafter [45], and its recent adaptation which can provide a realistic arrangement for a chromophore-embedded system: a detailed description of the adaptation of the CLP algorithm was recently published [19], and only an overview of the method is provided in the following section (see Fig. 3 ).

The first step of the process corresponds to the construction of a box containing one TPA-BMO molecule within a reasonably equilibrated butadiene-monomer bath. To this purpose, an orthorhombic unit cell containing one butadiene monomer at a quarter of the target density was designed and replicated $60 \times 6 \times 6$ times along the $\mathrm{x}-, \mathrm{y}$ - and z-axis, respectively (2160 monomers). A slice containing one TPA-BMO molecule was then stacked along this orthorhombic butadiene stacking. Periodic boundary conditions (PBC) are applied along the 3 directions. At this point the positions of the TPA-BMO atoms are fixed in order to preserve the internal structure of the fluorophore during the embedding procedure. In a second step, the system is progressively compressed to the target density $(\mathrm{d}=0.82)$ by a constant-velocity piston and the fluorophore is homogeneously embedded in the monomer bath. Then, in step 3, the chains are grown monomer by monomer with the CLP algorithm. At the end of the polymerization step, the chains are 20 to 25 monomers long which corresponds to a 80 to 100 carbon atoms in length. At that stage, the constraints on the TPA-BMO atomic positions are released and an equilibration / relaxation phase allows both the TPA-BMO molecule and the polymer matrix to structurally adjust to their new local environment. A representation of a simulation box corresponding to the TPA-BMO / polybutadiene system is provided in Fig. 3- Step 4. The different parameter sets for steps 1 to 3 (ensemble, temperature, friction parameter $\gamma$ of the Langevin thermostat, piston velocity, periodic boundary conditions PBC, time steps and simulation times) are the same as those provided in Le Bras et al [19]. 
Finally, the production phase is carried out during $1 \mathrm{~ns}$ in the NVT ensemble with a time step of $1 \mathrm{fs}$, using a Langevin thermostat.

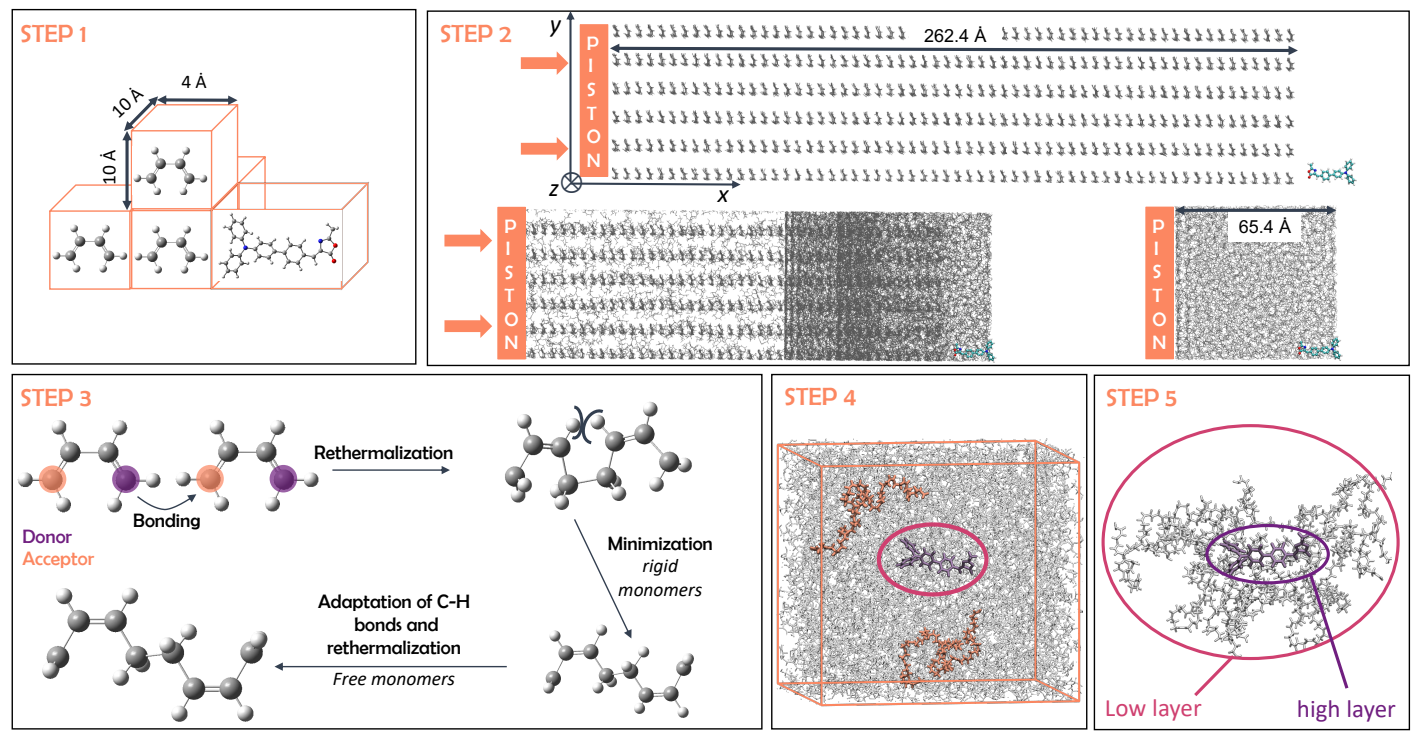

Figure 3: Computational process to study the optical properties of TPA-BMO in a polymer matrix. Step 1: simulation box building with a fixed TPA-BMO molecule in contact with an orthorhombic butadiene stacking. Step 2: compression of the system to the target density by a constant-velocity piston. Step 3: chain growth, monomer by monomer, with the CLP algorithm [45]. Step 4: TPA-BMO / polybutadiene simulation box (TPA-BMO and some polybutadiene chains are highlighted, in purple and orange respectively). The positional constraints on TPA-BMO are released for the equilibration and production runs. Step 5: definition of the high and low layers used for the QM/QM' calculations.

Then, ten snapshots are extracted at regular time steps $(0.1 \mathrm{~ns})$ along the trajectory. For each snapshot, the TPA-BMO molecule and the polymer chains with at least one atom localized at a distance smaller than 3.5 $\AA$ of any atom of the fluorophore, are selected (see Fig. 3 - Step 5). A comparison of different strategies for calculating the absorption properties of the TPA-BMO / polybutadiene system is presented and discussed in the Supporting Information section (Table S5). This study leads us to consider the ONIOM QM/QM' methodology within the polarisation embedding framework. The QM and QM' levels are respectively based on M06-2X/6-311+G(d,p) and HF/3-21G. The model region (or HL region) corresponds to the TPA-BMO molecule while the selected polymer chains are described at the low QM' level. 


\section{Results and Discussion}

\subsection{Properties of TPA-BMO in solution}

\subsubsection{Electronic structures and optical properties}

We have first determined the ground state structure of TPA-BMO in three different solvents (DXN, TOL, THF) relying on DFT with PCM model. The TPA-BMO molecule presents two isomers, $\mathrm{Z}$ and E, as shown in Fig.11. For the three solvents, we have systematically found that the value of the Gibbs free energy for the $\mathrm{Z}$ isomer is smaller than the $\mathrm{E}$ one by $0.15 \mathrm{eV}$. The Boltzmann population ratio for $\mathrm{Z}: \mathrm{E}$ being close to $99: 1$ and no Z-E photoisomerisation being experimentally observed, [8] all subsequent studies will be carried out on the Z isomer $\left(\phi_{B M O 2}=0^{\circ}\right)$.

Two torsional motions are possible along the $\phi_{D A}$ and $\phi_{B M O 1}$ dihedral angles represented in Fig. 1. In DXN, the geometry optimization has revealed the existence of different conformers corresponding to $\left(\phi_{B M O 1}=1^{\circ}\right.$, $\left.\phi_{D A}=37^{\circ}\right)$ and $\left(\phi_{B M O 1}=-1^{\circ}, \phi_{D A}=143^{\circ}\right)$. These two conformers present the same value of the Gibbs free energy and the same absorption properties (see Fig. S7). Hence, in the following, we will focus our discussion on the description of the conformer characterized by $\phi_{B M O 1}=1^{\circ}$ and $\phi_{D A}=37^{\circ}$.

The comparison of selected structural parameters shows that the solvent has a negligible impact on the $\mathrm{S}_{0}$ geometry (see Table S6 in Supporting Information). Consequently, the absorption wavelength are very close with calculated $\lambda_{a b s}$ values respectively equal to 378,379 and $381 \mathrm{~nm}$ in DXN, TOL and THF.

The computed absorption spectrum in DXN is provided in Fig. 4 and one can note a nice reproduction of the experimental feature. The calculated maximum absorption wavelength $\lambda_{a b s}$ corresponds to the $\mathrm{S}_{0} \rightarrow \mathrm{S}_{1}$ transition and is characterized by a HOMO $\rightarrow$ LUMO electronic excitation. Fig. 4 shows that the HOMO is mainly delocalized on the TPA donor part while the LUMO is centered on the BMO acceptor moiety. Therefore, the maximum absorption band, characterized by a $\pi-\pi^{*}$ transition, presents a charge transfer (CT) character with the CT dipole moment represented in Fig. 4

To compute the emission properties, the structure of the $S_{1}$ state has been optimized. The comparison of the structural parameters of $\mathrm{S}_{1}$ with $\mathrm{S}_{0}$ (Table $\mathrm{S} 6$ in S.I.) shows that, for the three solvents, the $\mathrm{d}_{T P A}, \mathrm{~d}_{B M O 1}$ and $\mathrm{d}_{D A}$ distances are shorter in the excited state than in the ground state. This finding results in an overall decrease of the total length of the molecule with, for instance, $\mathrm{d}_{t o t}=13.06 \AA$ for $\mathrm{S}_{0}$ and $\mathrm{d}_{t o t}=13.02 \AA$ for $\mathrm{S}_{1}$ in DXN. Moreover, upon relaxation on the $\mathrm{S}_{1}$ state, the torsional angle between the donor and the acceptor moieties $\phi_{D A}$ decreases by about $20^{\circ}$. The structure of TPA-BMO is thus more planar for $\mathrm{S}_{1}$ than $\mathrm{S}_{0}$. Finally, for $\mathrm{S}_{1}$, this dihedral angle value depends on the solvent polarity with $\phi_{D A}=17^{\circ}, 16^{\circ}$ and $14^{\circ}$ for respectively DXN, TOL and THF. 


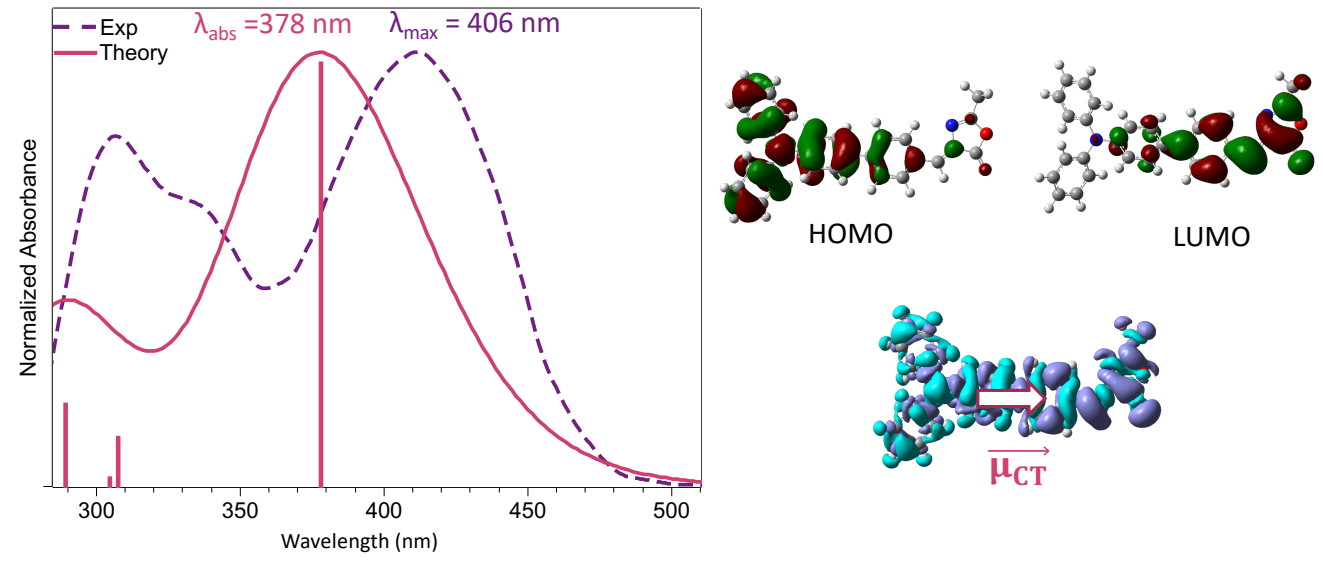

Figure 4: Calculated UV-Vis absorption spectrum of TPA-BMO in DXN (convolution with a Gaussian function, FMWH $=0.33 \mathrm{eV}$ ) and comparison with the experimental spectrum [8]. The orbitals involved in the $\lambda_{a b s}$ transition are represented, as well as the variation of the electronic density $\Delta \rho$ (dark blue: gain of electronic density; light blue: loss of electronic density).

It has been experimentally observed that the emission phenomenon undergoes a solvatochromic effect with $\lambda_{e m}=530,509$ and $576 \mathrm{~nm}$ in DXN, TOL and THF respectively [8]. We obtain the same behaviour with calculated emission wavelengths corresponding to 466, 469 and $505 \mathrm{~nm}$ in DXN, TOL and THF, which leads to a respective deviation from experiment of $0.32,0.21$ and $0.30 \mathrm{eV}$.

More generally, Tang et al [8]. have demonstrated that the absorption band is not solvatochrom as shown by Fig. 5(a). By considering 6 different solvents (DXN, TOL, THF, ACN but also DMSO and ACT), we obtain exactly the same trend with a quasi constant $\Delta \mathrm{E}_{\text {calc-exp }}$ deviation of $0.22 \mathrm{eV}$ (Fig. 5(b)). On the opposite, for the emission phenomenon, there is strong solvatochromic effect which is nicely reproduced by our calculations (Fig.5.(c)) with an average $\Delta \mathrm{E}_{\text {calc-exp }}$ deviation of $0.24 \mathrm{eV}$ (Fig.5.d)).

\begin{tabular}{c|cc|cccc}
\hline & \multicolumn{2}{|c|}{$\mathrm{D}_{C T}(\AA)$} & \multicolumn{4}{c}{$\mu_{C T}(\mathrm{D})$} \\
Solvent & $\mathrm{S}_{0} \rightarrow \mathrm{S}_{1}$ & $\mathrm{~S}_{1} \rightarrow \mathrm{S}_{0}$ & $\mathrm{~S}_{0} @ \mathrm{~S}_{0}$ & $\mathrm{~S}_{1} @ \mathrm{~S}_{0}$ & $\mathrm{~S}_{0} @ \mathrm{~S}_{1}$ & $\mathrm{~S}_{1} @ \mathrm{~S}_{1}$ \\
\hline DXN & 5.016 & 4.382 & 4.83 & 21.96 & 7.39 & 19.92 \\
TLN & 5.040 & 4.408 & 4.81 & 23.08 & 7.51 & 20.20 \\
THF & 5.263 & 4.671 & 5.16 & 23.13 & 9.03 & 23.12 \\
\hline
\end{tabular}

Table 2: Values of the $D_{C T}$ index observed upon the $S_{0} \rightarrow S_{1}$ and $S_{1} \rightarrow S_{0}$ transitions in DXN, TLN and THF. Values of the dipole moment $\mu$ in D calculated for the three different solvents for the $S_{i}$ state at the $S_{j}$ geometry $\left(\mathrm{S}_{i} @ \mathrm{~S}_{j}\right)$ with $i, j=0,1$.

To rationalize the non-solvatochromic (respectively solvatochromic) effect observed for the absorption (resp. emission) phenomenon, we have determined the $\mathrm{D}_{C T}$ index as well as the value of the dipole moment $\mu$ calculated for the $\mathrm{S}_{i}$ state at the $\mathrm{S}_{j}$ geometry $\left(\mu_{S_{i} @ S_{j}}\right)$ with $i, j=0,1$. First, we observe in Table 2 that for the three solvents, the $\mathrm{D}_{C T}$ index is large (about $5 \AA$ ), which is in agreement with the CT character of the 


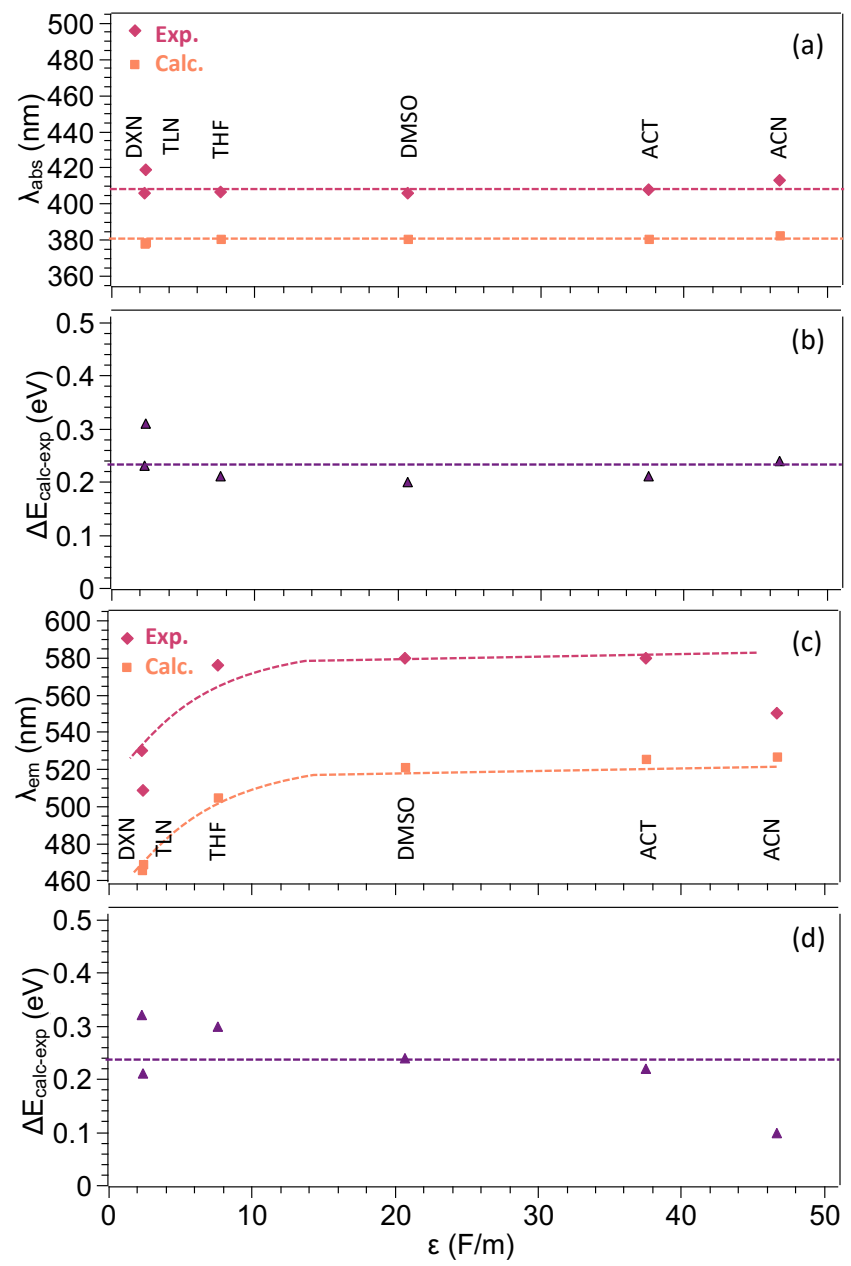

Figure 5: (a) Calculated $\lambda_{a b s}$ and experimental $\lambda_{\max }$ absorption wavelengths (in $\mathrm{nm}$ ) as a function of the solvent dielectric constant $\varepsilon$. (b) $\Delta \mathrm{E}_{\text {calc-exp }}$ deviation (in eV) calculated for the maximum absorption band as a function of $\varepsilon$. (c) and (d) Same as (a) and (b) for the emission wavelengths.

absorption band previously assigned via the frontier molecular orbital analysis. The same trends can be highlighted for the three solvents in the Franck-Condon region ( $\mathrm{S}_{0}$ geometry) with similar $\mathrm{D}_{C T}$ and dipole moment $\mu_{S_{0} @ S_{0}}$ values.

In addition, we observe that in DXN, the dipole moment increases from 4.83 D to $21.96 \mathrm{D}$ after the $\mathrm{S}_{0} \rightarrow \mathrm{S}_{1}$ excitation (in the Franck-Condon-FC- region). The relaxation on the first excited state then leads to a decrease of the dipole moment to the value of $\mu_{S_{1} @ S_{1}}=19.92 \mathrm{D}$. In THF, the dipole moment increases from $5.16 \mathrm{D}$ to 23.13 D upon the vertical electronic excitation and when the molecule relaxes on $\mathrm{S}_{1}$, contrary to DXN, the dipole moment remains constant $\left(\mu_{S_{1} @ S_{1}}=23.12 \mathrm{D}\right)$. This behaviour reflects the polarization of the electron density of the molecule by the solvent. Since THF is a more polar solvent, the charge separation within the 
TPA-BMO molecule is stabilized while relaxing on $\mathrm{S}_{1}$. Thus, the stabilization of the CT state decreases the deexcitation energy and red-shifts the fluorescence emission spectra.

This solvatochromic effect can be generally explained by the Lippert-Mataga equation derived from Onsager's reaction field theory [50]. As shown in Supporting Information, there is linear relation between the calculated Stokes shift $\Delta \bar{v}$ between the spectra peak frequency of emission and absorption, and the solvent polarity described with the quantity $\Delta f$ :

$$
\Delta f=\frac{\varepsilon-1}{2 \varepsilon+1}-\frac{n^{2}-1}{2 n^{2}+1}
$$

where $\varepsilon$ and $\mathrm{n}$ is the static dielectric constant and the optical refractive index of the solvent, respectively. This confirms that for TPA-BMO, the general solvent effect prevails: the emission wavelength increases with the solvent polarity.

\subsubsection{Modulation of the emission efficiency}

In solution, for low polar solvents, Tang et al. have observed a decrease of the fluorescence quantum yield due to the increase of the solvent polarity (see Table 1).

First, regarding the modulation of the emission properties, conical intersections (CI), regions of the potential energy surfaces (PES) where the ground state $\mathrm{S}_{0}$ and the excited states become degenerate, can play an important role regarding the non-radiative deactivation pathway. Hence, we have explored the topology of the PES of the low-lying states to investigate the existence of energetically accessible CI regions. The relative energy of the $S_{0}, S_{1}$ and $S_{2}$ states of TPA-BMO as a function of the dihedral angle $\phi_{D A}$ and $\phi_{B M O 1}$ is given in Fig. 6 for TPA-BMO in TLN. We remind here that no Z-E photoisomerization was experimentally observed [8]. Hence, the scan along the $\phi_{B M O 2}$ corresponding to the Z-E torsion has not been investigated.

For both energy profiles, we have scanned the torsional angle from $0^{\circ}$ to $180^{\circ}$ with a $10^{\circ}$ step, optimized the remaining structural parameters for the ground state $S_{0}$ and calculated the vertical excitation energy for $S_{1}$ and $S_{2}$ (solid lines). We have also calculated the same energy profile for $S_{1}$ (the $S_{1}$ state has been optimized) but the angle interval along the scan is $30^{\circ}$ (dotted lines). The structural and energetical parameters of the optimized $\mathrm{S}_{0}$ and $\mathrm{S}_{1}$ geometries (optimization carried out without any constraint) are also given ( $\mathrm{S}_{0, o p t}$ and $S_{1, \text { opt }}$ in Fig. 6. In the FC region, $S_{1}$ and $S_{2}$ states both present a CT character, the CT character of $S_{1}$ being more pronounced than $S_{2}$. For instance, the $D_{C T}$ index value is respectively 5.04 and $4.06 \AA$ for $S_{1}$ and $\mathrm{S}_{2}$ with a CT from TPA to BMO. Along the PES presented in Fig. 6 the CT character of both the $\mathrm{S}_{1}$ and

$S_{2}$ states is preserved (a detailed analysis of the nature of $S_{1}$ and $S_{2}$ for the $S_{0, o p t}, S_{1, o p t}, S_{1, D A-b a r r i e r}$ and $\mathrm{S}_{1, B M O 1-b a r r i e r}$ structures is provided in Supporting Information). 
The analysis of these energy profiles reveals that there is no conical intersection in the considered regions where the excited states of TPA-BMO can be efficiently deactivated to the ground state $\mathrm{S}_{0}$. Consequently, for low polar solvents, the modulation of the emission efficiency can not be explained by rapid radiationless decay due to electronic energy funnels.
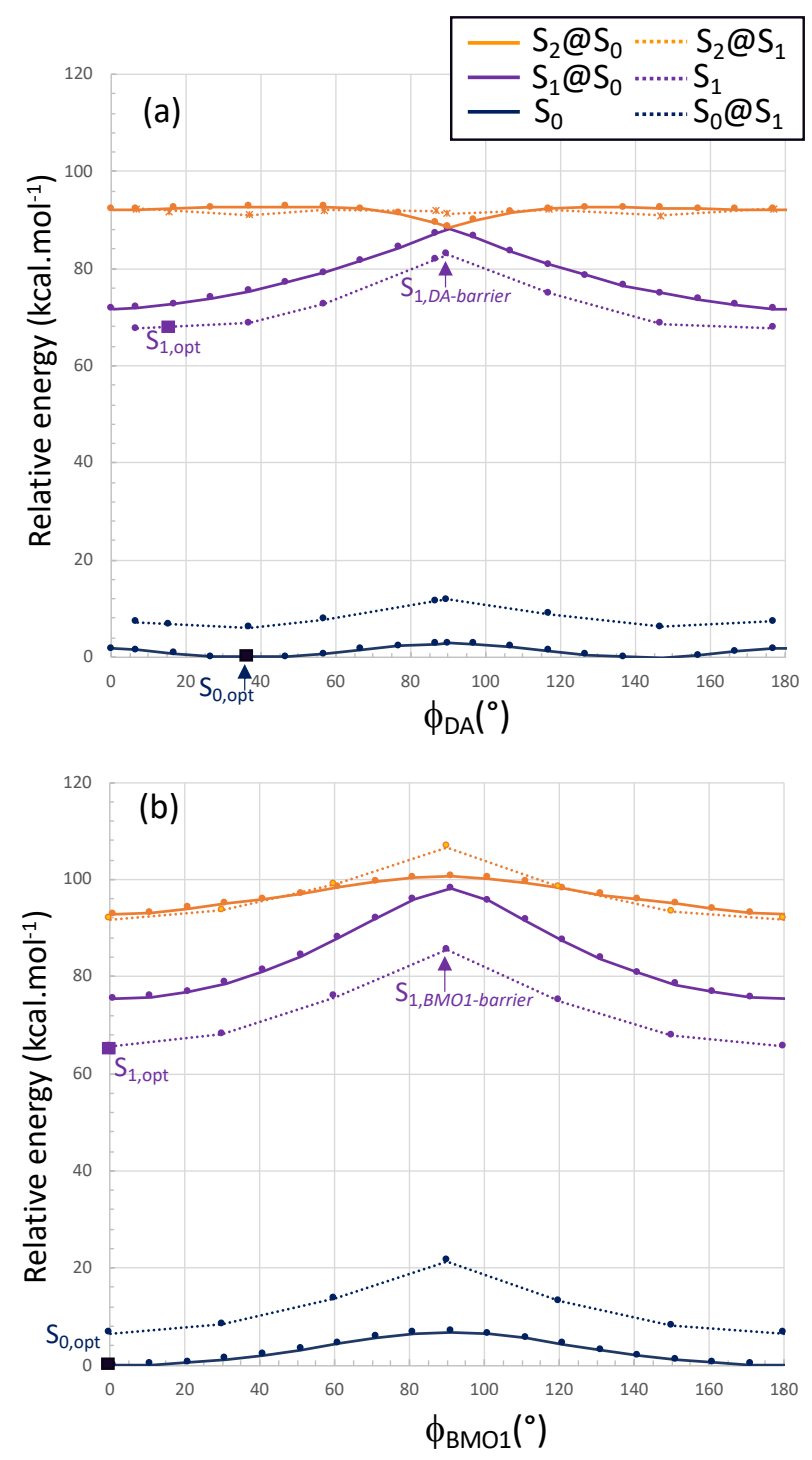

Figure 6: Relative energy in kcal.mol ${ }^{-1}$ of the $\mathrm{S}_{0}, \mathrm{~S}_{1}$ and $\mathrm{S}_{2}$ states as a function of the $\phi_{D A}$ (a) and $\phi_{B M O 1}$ (b) dihedral angle (TLN solvent). For fixed values of $\phi_{D A}$ and $\phi_{B M O 1}$ (step range $=10^{\circ}$ ), the ground state structure $S_{0}$ is optimized and the vertical excitation energies $S_{1} @ S_{0}$ and $S_{2} @ S_{0}$ are given (solid lines). The first excited state $S_{1}$ is also optimized for fixed values of $\phi_{D A}$ and $\phi_{B M O 1}$ (step range $=30^{\circ}$ ) and the vertical projected energies of $S_{0}$ and $S_{2}\left(S_{0} @ S_{1}\right.$ and $\left.S_{2} @ S_{1}\right)$ are also given (dotted lines). $S_{0, o p t}$ and $S_{1, o p t}$ refer to the GS and first excited state optimized structure, without any constraint. The energy reference corresponds to $\mathrm{S}_{0, o p t}$. 
Non radiative mechanisms can also arise from the transfer of the electronic excitation energy to low-frequency vibrational modes, these are the RIR and RIM mechanisms described in introduction. To investigate the role of the electronic to vibrational energy conversion on the modulation of emission efficiency, we have computed the HR factors and reorganization energies in TLN, DXN and THF.
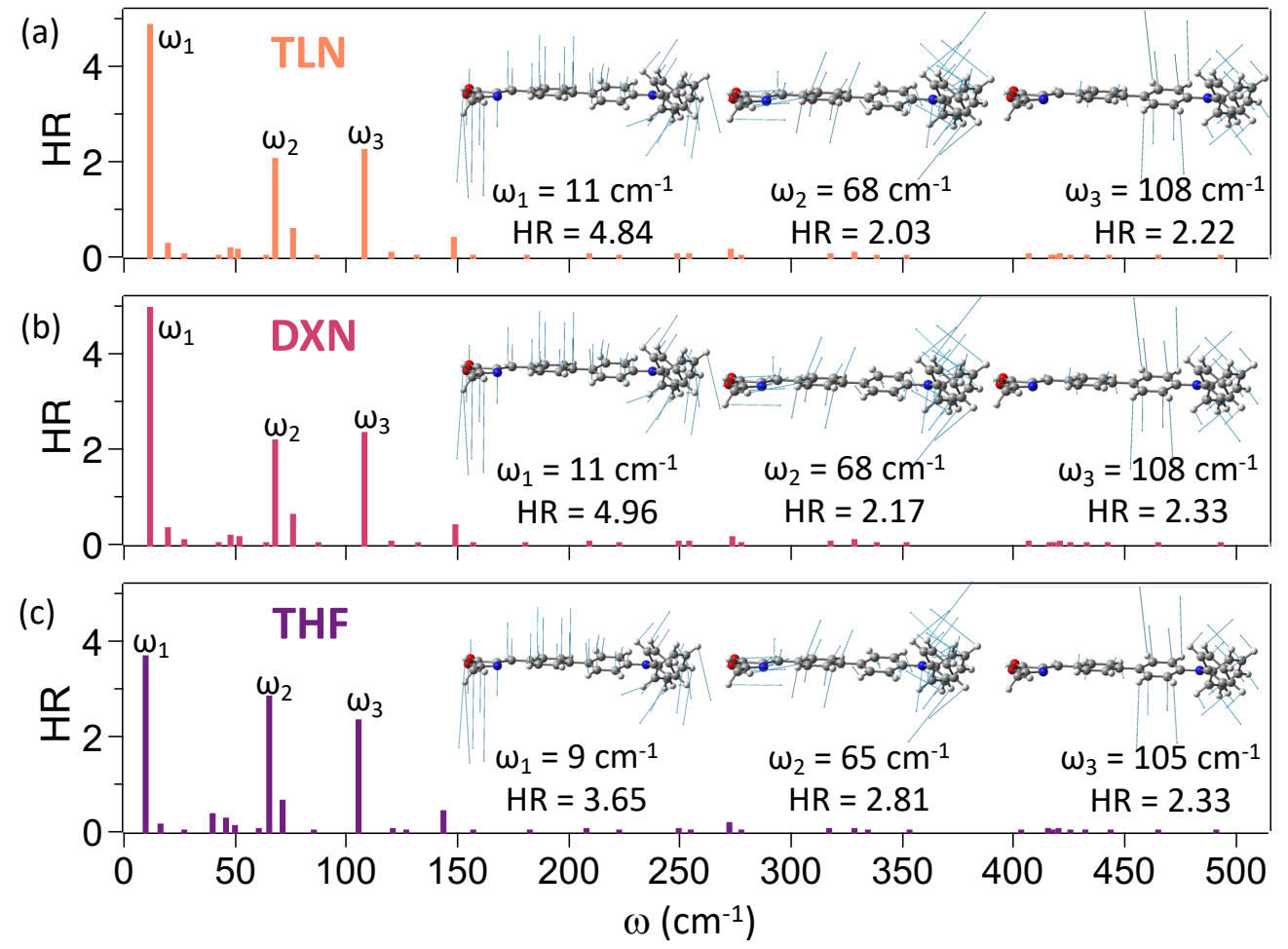

Figure 7: Calculated HR factors versus the normal-mode wave numbers $(\omega)$ in the $S_{1}$ state for TPA-BMO in (a) TLN, (b) DXN and (c) ACN. Vibrational modes yielding the largest HR factors are also represented.

As mentioned in the section 2.1. HR factors can be used to qualitatively assess the quantum fluorescence yield, $\Phi_{F}$ with $\Phi_{F}=k_{r} /\left(k_{r}+k_{n r}\right), k_{r}$ the radiative decay rate and $k_{n r}$ the non-radiative decay rate. Indeed, $k_{n r}$ directly depends on the Internal Conversion (IC) rate, in the absence of intersystem crossing such as this is the case for TPA-BMO. Besides, the IC rate directly relies on HR factors [29, 51]. In this context, Dua et al have shown that there is a direct quantitative relationship between the HR factor values, the IC rate and the non-radiative decay rate constant [29]. They have shown that the non-radiative decay process and the decrease of $\Phi_{F}$ are directly linked to the presence of low-frequency modes with large HR factors. The photophysical energy dissipation caused by vibrational motions can then be evaluated by the calculation of the reorganization energy (Eq. 2). In previous works dedicated to the study of other AIEgen families in solution, crystal and aggregate, the qualitative correlation between reorganization energy and the quenching of the 
emission process has been demonstrated [14, 15, 16, 51] and we propose to use the same strategy in the present study. More quantitative studies can be considered for molecules in solution[52] but cannot be undertaken for molecules within an aggregate or dispersed in a polymer matrix. Since we are interested in the comparison of the TPA-BMO behavior in these three environments, we have calculated the total reorganization energy $\mathrm{E}_{\text {reorg }}$ and qualitatively correlated the calculated value with the emission efficiency.

In this context, the $\mathrm{E}_{\text {reorg }}$ value is 2880,2884 and $3080 \mathrm{~cm}^{-1}$ in TLN, DXN and THF, respectively, while $\Phi_{F}$ is $0.68,0.62$ and 0.51 . Hence, the larger the $\mathrm{E}_{\text {reorg }}$ value is, the smaller $\Phi_{F}$ is, as already observed in previous works where the photophysical energy dissipation caused by vibrational motions is responsible for the emission quenching [14, 15, 16]. In more details, at low frequencies, three vibrational modes present important HR factor values (shown in Fig. 77). The first mode, $\omega_{1}$, corresponds to a motion outside the plane of the entire molecule. The HR value associated with this mode decreases with the solvent polarity. The second mode, $\omega_{2}$, corresponds to a BMO scissor motion centered on the angle $\theta_{2}$ as well as a balance movement of the BMO phenyl cycle. In that case, the HR factor increases significantly as the solvent polarity increases (HR $=2.03$ and 2.81 in TLN and ACN respectively). Finally, $\omega_{3}$ corresponds mainly to a rotation of the TPA phenyl groups and there is no dependence of the HR factor on the solvent polarity.

\subsection{Properties of TPA-BMO within an aggregate}

\subsubsection{Structural and optical properties}

Along the $10 \mathrm{~ns}$ simulation, the aggregate encompassing 30 TPA-BMO molecules represented in Fig. 2 is stable, there is no splitting into smaller clusters. The analysis of this trajectory reveals that we can distinguish two different regions within the aggregate. On the one hand, the center of the aggregate encompasses TPA-BMO molecules that are not in contact with the solvent molecules and that do not present specific order. On the other hand, for the periphery of the aggregate, the molecules form "head-to-head" dimers, thanks to $\pi$ - $\pi$ stacking interactions as shown in Fig. 2r(c). Within these dimers, the distance between two TPA-BMO molecules is $\sim 4.1$ A. Moreover, there are strong interactions between the water solvent molecules and TPA-BMO. The analysis of the radial distribution functions (Fig. S6) shows the formation of three hydrogen bonds between the oxazole group and three different $\mathrm{H}_{2} \mathrm{O}$ molecules.

We have compared the time evolution of the structural parameters for (1) an isolated molecule in water solution, (2) a molecule at the center and (3) 8 molecules at the periphery of the aggregate, along a $10 \mathrm{~ns}$ trajectory. We did not observe important variation of the bonds and angles when comparing the three systems (Table S8). On the opposite, the environment has a strong impact on the dihedral angles $\phi_{D A}$ and $\phi_{B M O 1}$. 


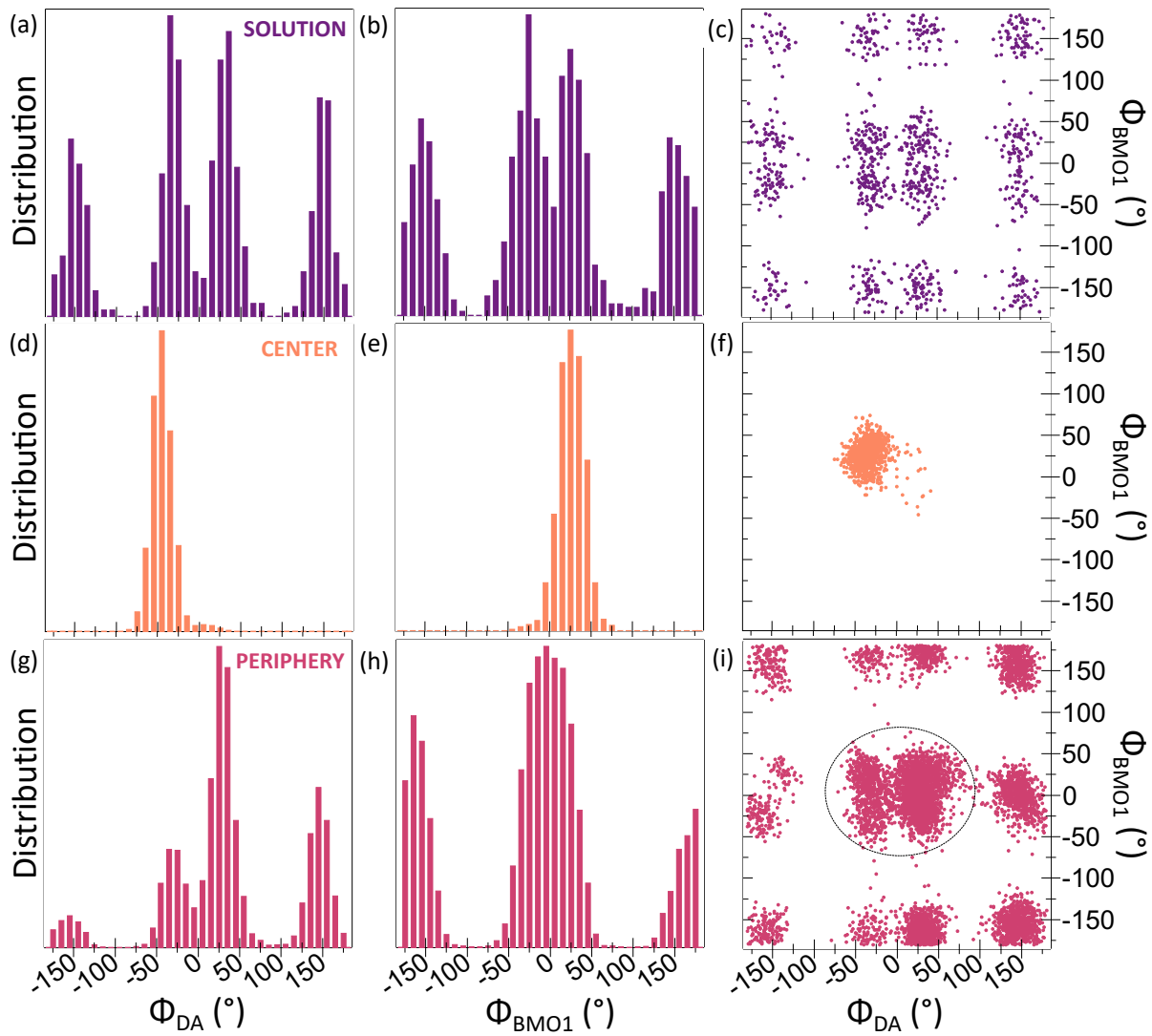

Figure 8: (a) Normalized distribution of the dihedral angle $\phi_{D A}$, (b) normalized distribution of the dihedral angle $\phi_{B M O 1}$ and (c) representation of value couples $\left(\phi_{D A}, \phi_{B M O 1}\right)$ for an isolated molecule of TPA-BMO in water solution along a $10 \mathrm{~ns}$ trajectory. (d), (e), (f) Same information for one molecule localized at the center of the aggregate. (g), (h), (i) Same information for 8 molecules localized on the edge of the aggregate.

For a free molecule in solution, Fig. 8 (a) and (b) show that $\phi_{D A}$ dihedral angles can adopt four distinct values centered at $\sim \pm 35^{\circ}$ and $\sim \pm 150^{\circ}$ while for $\phi_{B M O 1}$, the preferential angle values are $\sim \pm 25^{\circ}$ and $\sim \pm 150^{\circ}$. This leads to the existence of different conformers while for the molecule localized at the center of the aggregate, the angle distributions are logically narrower and only one conformer is observed (Fig. 8 (f)). This loss of flexibility is due to the steric hindrance imposed by the rest of the aggregate. Indeed, the values of angle $\phi_{D A}$ is between $-70^{\circ}$ and $-10^{\circ}$, with a maximum of the distribution obtained for $-35^{\circ}$ (Fig. 8 8 d)). For $\phi_{B M O 1}$, the distribution represented in Fig. 8 (e) peaks at a $\sim 25^{\circ}$ angle value, with the torsion angle ranging from $-5^{\circ}$ to $65^{\circ}$. Finally, the molecules located at the periphery of the aggregate are logically more flexible than those at the center: the standard deviation of $\mathrm{d}_{t o t}$ is respectively $0.14 \AA$ and $0.03 \AA$. In addition, as depicted in Fig. $8 \mathrm{~g}$ g) and (h), the dihedral angle $\phi_{D A}$ can adopt 4 different values $\left(-150^{\circ},-25^{\circ}, 25^{\circ}, 150^{\circ}\right)$ while the $\phi_{B M O 1}$ distribution shows three possible values $\left(-170^{\circ}, 0^{\circ}, 170^{\circ}\right)$. By plotting the correlation between these two angles (see Fig. 8(i)), a large number of conformers can be observed and the black dotted circle on Fig. 
8 (i) encompasses half of the snapshots extracted along the trajectory. Globally, the most probable value of $\phi_{D A}$ is $\pm 37^{\circ}$ for an isolated molecule in solution, this value slightly decreases to $35^{\circ}$ for a molecule at the center of the aggregate and drops to $25^{\circ}$ for the molecules at the periphery (Fig. $8(\mathrm{a}, \mathrm{d}, \mathrm{g}$ )). A similar trend is observed for $\phi_{B M O 1}$ (Fig. 8 b, e, h)). There is thus a flattening of the molecular structure from the center to the periphery of the aggregate. This can be explained by the existence of $\pi-\pi$ interactions between TPA-BMO molecules at the edge of the aggregate as shown in Fig. 2(r).

(a)

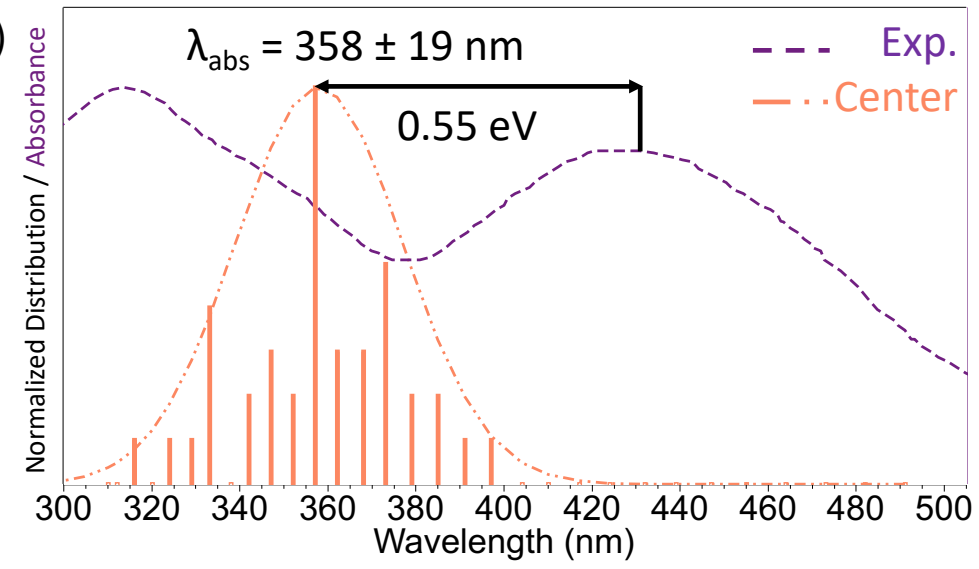

(b)

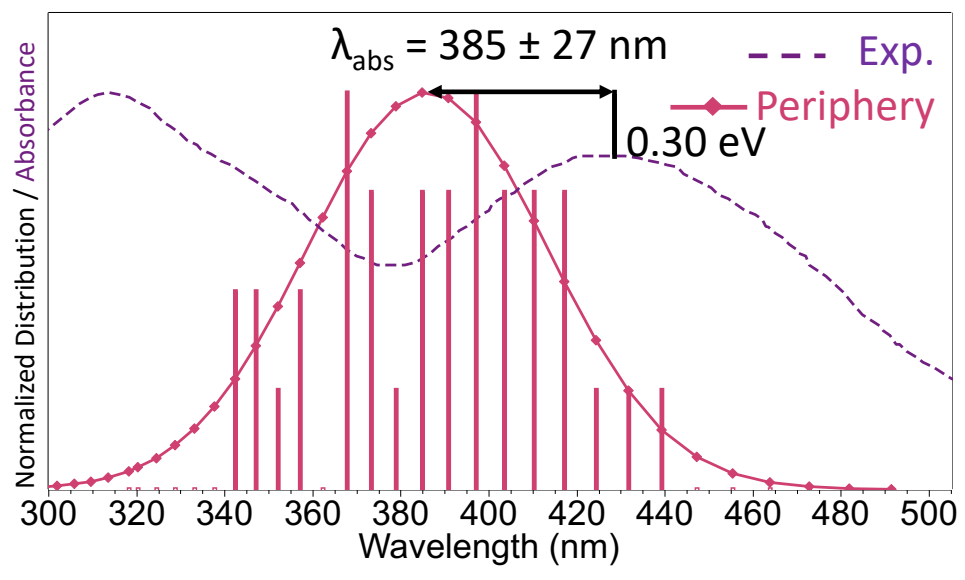

Figure 9: Histograms of the normalized distribution of the maximum absorption wavelength $\lambda_{a b s}$ in $\mathrm{nm}$ (bin sizes: $0.10 \mathrm{eV}$ ) and comparison with the experimental UV-Vis absorption spectrum (normalized absorbance) [9]. Distributions calculated for (a) molecules located at the center of the aggregate and (b) molecules located at the periphery (in each case, 40 different structures are considered, see text).

This variation of flexibility has an impact on the calculation of the absorption spectra as shown in Fig. 9 First, to calculate the absorption spectrum for the molecules at the center of the aggregate, we have extracted 20 different snapshots (every 50 ps) and for each snapshot, we have identified 2 molecules localized at the center. We have thus calculated 40 different absorption spectra and for each calculation, we have considered 
the excitation energy corresponding to the absorption wavelength with the largest $f$ value, $\lambda_{a b s}$. We have then plotted the histograms corresponding to the $\lambda_{a b s}$ normalized distribution using a bin size of $0.1 \mathrm{eV}$ and finally fitted this distribution with a Gaussian function. Fig. 9(a) shows that the distribution is centered at $\lambda_{a b s}=358 \mathrm{~nm}$ with a standard deviation $\sigma=19 \mathrm{~nm}$. The deviation from experiment is large as shown in Fig. 9 with an energy difference of $0.55 \mathrm{eV}$ between the experimental and calculated maximum absorption band.

For the molecules located at the periphery of the aggregate, we have followed the same strategy and calculated 40 different absorption spectra based on the same 20 snapshots and randomly selecting 2 molecules at the edge. The higher flexibility of the molecules at the periphery of this aggregate leads to a broader distribution with a standard deviation $\sigma=27 \mathrm{~nm}$. Besides, the maximum of the distribution $\left(\lambda_{a b s}=385 \mathrm{~nm}\right)$ is red-shifted compared to the molecules at the center, this is a direct consequence of the flattening of the molecular structure, as previously discussed. Moreover, our methodological study provided in Table S4 has shown that taking into account explicit $\mathrm{H}_{2} \mathrm{O}$ molecules in the high level region of the ONIOM QM:QM' calculations is of high importance. Indeed, for a given snapshot, with (respectively without) explicit $\mathrm{H}_{2} \mathrm{O}$ molecules, the computed $\lambda_{a b s}$ value is $417 \mathrm{~nm}$ (resp. $374 \mathrm{~nm}$ ),ds a detailed comparison is provided and discussed in Fig. S4 in Supporting Information. Thus, the distribution of the $\lambda_{a b s}$ values calculated for the molecules at the periphery of the aggregate in presence of explicit water molecules nicely reproduces the experimental absorption band with a deviation of $0.30 \mathrm{eV}$ as shown in Fig. 9 b). In the following, we will investigate the emission properties of a molecule loacted both at the periphery and at the center.

\subsubsection{Emission properties and modulation of quantum yields}

The geometry of the first excited state has been optimized for a molecule at the periphery and at the center of the aggregate. First, for a molecule located at the edge, the relaxation on $S_{1}$ leads to (i) a slight contraction of the molecule with a decrease of the $\mathrm{d}_{t o t}$ distance, (ii) a decrease of the $\mathrm{d}_{B M O 1}$ distance, (iii) a decrease of $\phi_{D A}$ by about $15^{\circ}$, and (iv) a large modification of the TPA moiety with a decrease of the $\phi_{T P A}$ torsional angle of $34^{\circ}$ and a decrease of the $\mathrm{d}_{D A}$ distance by $\sim 0.1 \AA$ (Table S10). The modifications (i) to (iii) have also been observed for solvated molecules, contrary to the observation (iv). The structural modification observed for the TPA group is certainly due to the strong distorsion of this moiety for the snapshot extracted from the MD simulation.

For a molecule located at the center of the aggregate, upon relaxation on $S_{1}$, the major structural evolution is the decrease of the $\phi_{D A}$ angle. However, contrary to solvated molecules or molecules located at the edge of the aggregate, the variation is much smaller (decrease of $6^{\circ}$ ) compared to a decrease of 15 to $20^{\circ}$ for the other environments. This is due to the strong steric constraints imposed to the molecule located at the center by the neighboring molecules. 
For a molecule located at the periphery, the strong structural modifications observed upon relaxation on $\mathrm{S}_{1}$ lead to a large Stokes shift. The calculated emission energy is $550 \mathrm{~nm}$ and we nicely reproduce the experimental emission wavelength ( $\lambda_{e m}=573 \mathrm{~nm}$, Tab. 1) with a deviation of $0.09 \mathrm{eV}$. On the opposite, the Stockes shift is much smaller for a molecule located at the center, the calculated emission wavelength reaching $421 \mathrm{~nm}$.

In the aggregate, a modification of the potential energy surfaces presented on Fig. 6leading to the appareance of $\mathrm{S}_{0} / \mathrm{S}_{1}$ intersection would enhance the nonradiative decay and can not explain the AIE effect observed by Tang et al. [8, 9]. Therefore, the modulation of the emission properties of TPA-BMO observed within the aggregate may first be explained by a modification of the energy dissipation due to the vibrational modes. Hence, the vibrational spectrum of the $S_{1}$ structure for a molecule at the periphery of the aggregate is compared to the one obtained in solution. One has to note that is not possible to compute the HR factors within the ONIOM calculation framework. Fig. 10 reveals a strong modification of the overall low-frequency vibrational spectrum for both a molecule located at the center and at the periphery. Moreover, the inspection of all the molecular vibrational modes at low frequencies $\left(0\right.$ to $\left.160 \mathrm{~cm}^{-1}\right)$ reveals that the three modes modes mostly contributing to the energy dissipation in solution (Fig. 7) can not be retrieved in the aggregated environment. We remind here that these three vibrational modes are (1) a motion outside the plane of the entire molecule, (2) a BMO scissor motion centered on the angle $\theta_{2}$ as well as a balance movement of the BMO phenyl cycle and (3) a rotation of the TPA phenyl groups. For the aggregate, the vibrational modes presenting some similarities with these three modes are provided but globally, one can note that the modes involving the TPA moiety are hindered in the aggregate. We thus expect that no vibrational mode should be efficiently involved in the nonradiative decay process. 

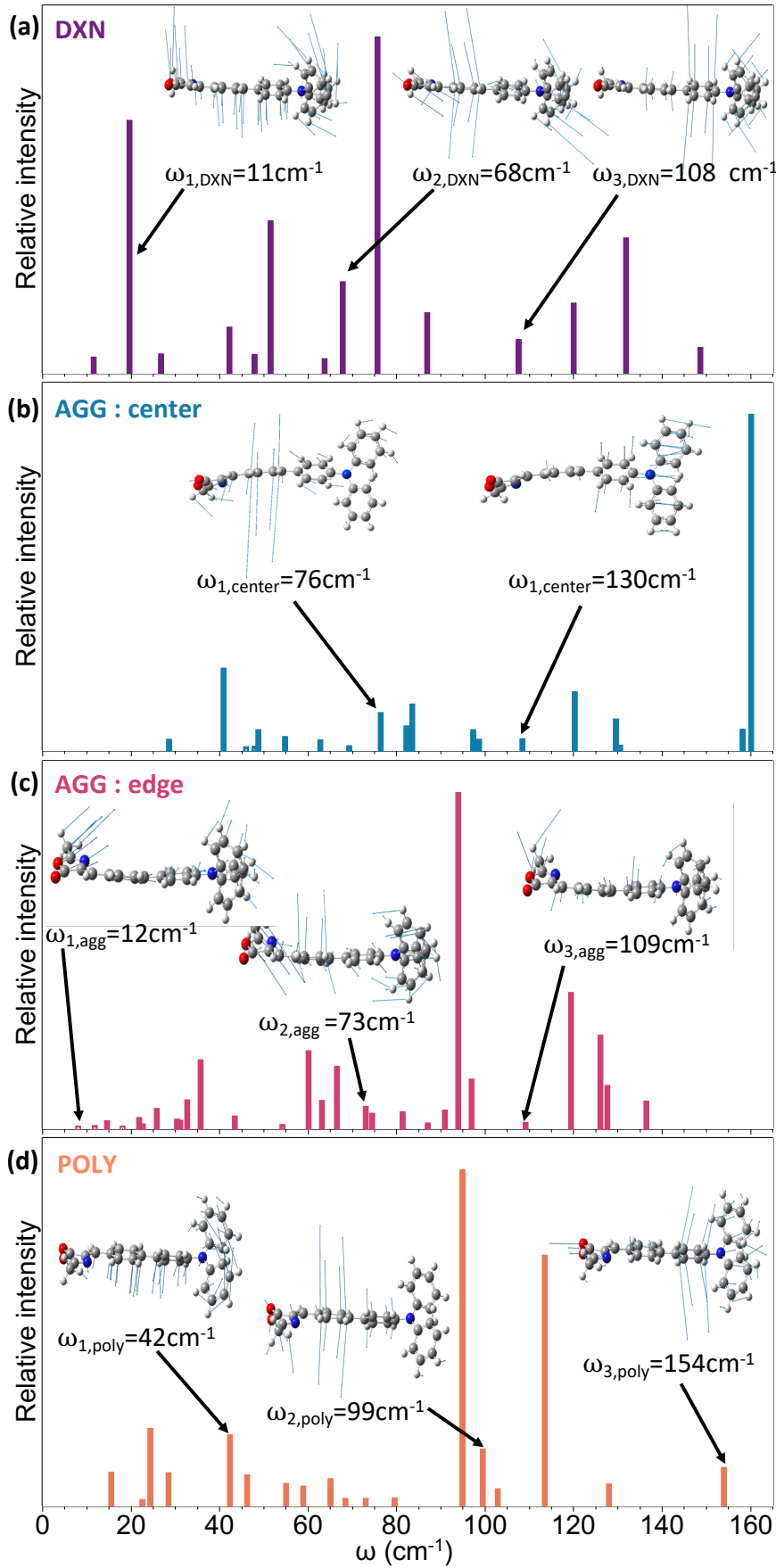

Figure 10: Low-frequency vibrational spectra $\left(0-160 \mathrm{~cm}^{-1}\right.$ region) calculated for the $S_{1}$ state of TPA-BMO in (a) DXN, (b) located at the center of the aggregate, (b) located at the periphery of the aggregate and (d) within the polymer matrix. The vibrational modes corresponding to large HR values in solution are given, and the modes presenting similarities in the aggregate or within the polymer matrix are depicted. 
Another phenomenon can explain the modulation of $\Phi_{F}$, namely the possible energy loss through excitation energy transfers between two TPA-BMO molecules forming a dimer at the periphery of the aggregate (Fig. 2). The value of the excitonic coupling $\mathrm{J}_{i j}$ between 2 molecules $i$ and $j$ in a dimer[53, 54] reaches an average value of $\mathrm{J}_{i j}=60 \mathrm{meV}$, which is considered as a "medium" coupling. Since the excitonic coupling always increases the non-radiative decay constant,[54] the molecular arrangement within the aggregate should promote the non-radiative relaxation pathway.

Consequently, there is competition between two antagonistic effects, (1) the absence of vibrational mode potentially contributing to the energy dissipation and (2) the existence of "medium" excitonic couplings leading to moderate energy loss. for the molecules located at the edge of the aggregate. Overall, the observed AIE effect can thus be interpreted by the predominant role of the first effect: the restriction of intramolecular vibration (RIV) mechanism should open the radiative decay channels.

\subsection{Properties of TPA-BMO within the polymer matrix}

\subsubsection{Structural and absorption properties}

The structural parameters of TPA-BMO embedded in the polybutadiene polymer matrix are analyzed along the 1 ns trajectory. Fig. 11.a) and (b) show the distribution of the dihedral angles $\Phi_{D A}$ and $\Phi_{B M O 1}$. These distributions respectively peak at $-65^{\circ}$ for $\Phi_{D A}\left(-35^{\circ}\right.$ in solution, see Fig. 8 (a)) and $40^{\circ}$ for $\Phi_{B M O 1}\left(-25^{\circ}\right.$ in solution, Fig. 8(b)). Thus, a global loss of planarity of the fluorophore is observed when going from the solution to the polymer matrix. More precisely, the dihedral angle $\Phi_{D A}$ preferentially adopts the $-65^{\circ}$ and $-125^{\circ}$ values, while the dihedral $\Phi_{B M O 1}$ ranges between $-55^{\circ}$ and $40^{\circ}$.
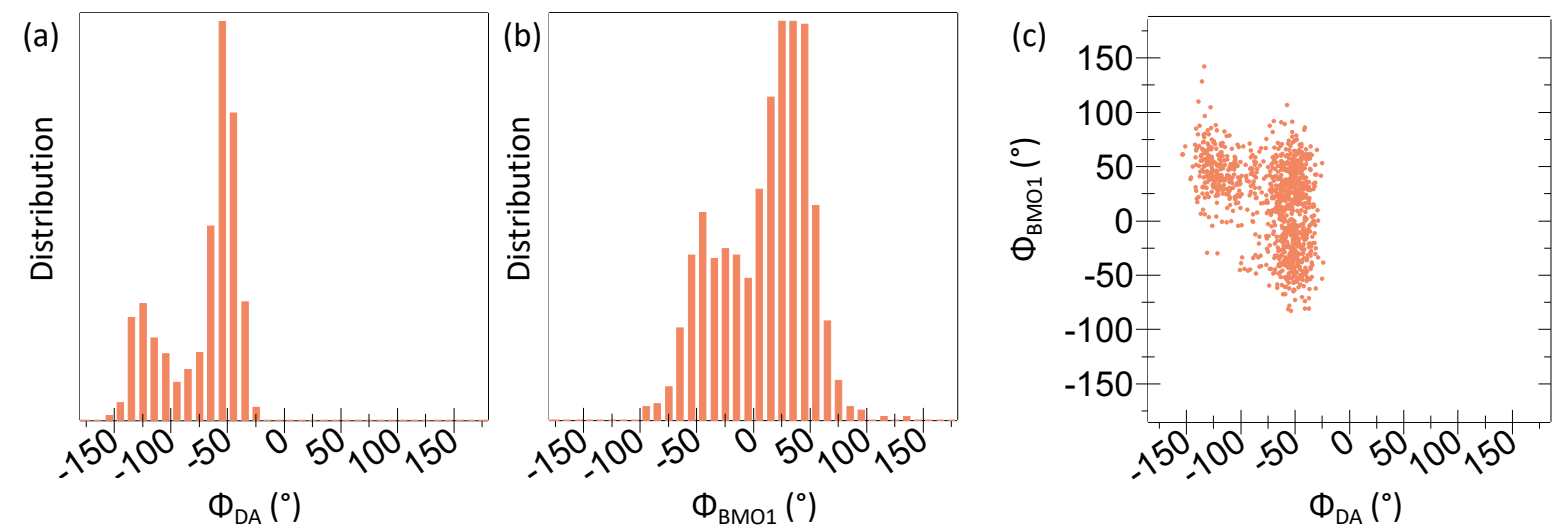

Figure 11: (a) Normalized distribution of the dihedral angle $\phi_{D A}$, (b) normalized distribution of the dihedral angle $\phi_{B M O 1}$ and (c) representation of value couples $\left(\phi_{D A}, \phi_{B M O 1}\right)$ for a TPA-BMO molecule embedded in a polybutadiene matrix along a 1 ns trajectory. 
As depicted in Fig. 11.c), two different conformers can be identified and consequently, there is a loss of flexibility of the TPA-BMO embedded in the polymer matrix compared to the solution (see Fig. 8. $(\mathrm{c})$ ). Taking into account both the loss of flexibility and planarity of the fluorophore allows us to highlight the mechanical effect of the polymer matrix.

To investigate the optical properties, 10 different snapshots encompassing a TPA-BMO molecule and its its closest neighboring polymer chain are extracted along the $1 \mathrm{~ns}$ trajectory (every $0.1 \mathrm{~ns}$ ). For one snapshot, we have compared the impact of the environment on the computed excitation energies. When TPA-BMO is considered without the polymer chains, the calculated absorption wavelength is $\lambda_{a b s}=381 \mathrm{~nm}$. When the polymer chains are taken into account within a ONIOM QM/QM'-PE strategy, we observe a blue shift of $0.06 \mathrm{eV}$ with a computed $\lambda_{a b s}$ value equals to $374 \mathrm{~nm}$ (also see Table S5). Consequently, the presence of the polybutadiene matrix has also an electronic impact on the absorption properties of the fluorophore.

In this context, for the ten snapshots, the computed $\lambda_{a b s}$ range from $335 \mathrm{~nm}$ to $409 \mathrm{~nm}$ with an average value of $350 \mathrm{~nm}$ and a standard deviation of $\sigma=23 \mathrm{~nm}$. Hence, there is a large distribution of the computed absorption properties as shown in Fig. 12

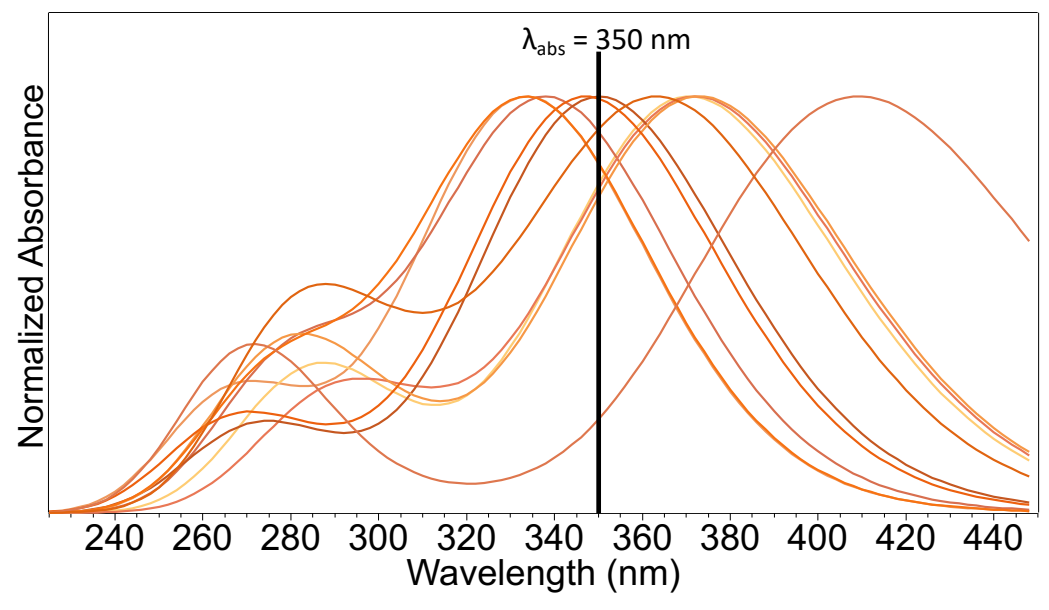

Figure 12: Comparison of the convoluted absorption spectra calculated for the ten snapshots extracted along the $1 \mathrm{~ns}$ simulation of TPA-BMO embedded in a polybutadiene matrix. Calculated excitation energies are convoluted with a Gaussian function $(F W M H=0.33 \mathrm{eV})$. The average value of $\lambda_{a b s}$ is also provided.

\subsubsection{Emission properties and modulation of quantum yields}

The geometry of the first excited state has been optimized for a TPA-BMO molecule surrounded by its closest neighboring polymer chain. The relaxation on $\mathrm{S}_{1}$ leads to a slight extension of the molecule with an increase of $\mathrm{d}_{t o t}$ compared to the $\mathrm{S}_{0}$ structure (Table $\mathrm{S} 11$ ) but also a flatening of the BMO moiety with a small decrease 
of both $\phi_{B M O 1}$ and $\phi_{B M O 2}$ dihedral angles. More importantly, a decrease of the $\phi_{D A}$ torsional angle is observed with a value of $-55^{\circ}$ for $S_{0}$ and $-23^{\circ}$ for the $S_{1}$ optimized structure.

The calculated emission energy is $437 \mathrm{~nm}$ and we nicely reproduce the experimental emission wavelength $\left(\lambda_{\text {em }}=499 \mathrm{~nm}\right.$, Tab. 1) with a deviation of $0.35 \mathrm{eV}$. This blue shift in the emission wavelength relative to the solution or the aggregate may be related to the structural differences observed. Indeed, when comparing the $\mathrm{S}_{1}$ structures, the one obtained within the polymer matrix is the least planar $\left(\phi_{D A}=-23^{\circ}\right)$ when compared to the solution or the aggregate $\left(\phi_{D A}=17^{\circ}\right.$ and 13 , respectively). As previously observed for the ground state, this is due to the mechanical constraints imposed by the polymer matrix which leads to a conjugation loss and a blue-shift of the emission energy.

Fig. 13 compares the calculated and experimental absorption and emission spectra obtained for TPA-BMO in a dioxane solution (Fig. 13,a)) and within the polybutadiene matrix (Fig. 13.b)). Experimentally, a blue shift of $0.16 \mathrm{eV}(35 \mathrm{~nm})$ is observed between the emission wavelength, $\lambda_{e m}=534 \mathrm{~nm}$ in DXN[8] and $\lambda_{e m}=499 \mathrm{~nm}$ in the polymer matrix [11]. Our calculations provide a blue shift of $0.18 \mathrm{eV}(29 \mathrm{~nm})$. Therefore, the calculation protocol that we have designed and applied to this complex environment is capable of reproducing the trend observed experimentally between the DXN solution and the polybutadiene polymer matrix.

To investigate the emission efficiency within the polybutadiene matrix, the frequencies of the first excited state are computed and compared to those obtained in solution (Fig. 10). It was possible to identify the same vibrational modes for which the values of the HR factors were large in solution, namely (1) the same out-of-plane vibration motion at $\omega_{1}=42 \mathrm{~cm}^{-1}$, (2) the pendulum motion of the TPA phenyl cycles (even if the amplitude is greatly attenuated) along with the slightly modified vibration of the BMO methyl group and the BMO phenyl rotation (with an increase of the amplitude) at $\omega_{2}=99 \mathrm{~cm}^{-1}$, and (3) the rotation of the TPA

phenyl groups at $\omega_{3}=154 \mathrm{~cm}^{-1}$. The fact that we have been able to retrieve the same vibrational modes as the ones previously identified in solution (DXN) suggests a similar resulting emission efficiency within the matrix. The dispersion of the energy during the relaxation of the excited state should thus be of the same order of magnitude between those two phases (DXN and polybutadiene) and we expect $\Phi_{F}$ within the polymer matrix to be at least equal to the one obtained in solvent with a low polarity. This in agreement with the experimental observations since there is no emission quenching when TPA-BMO is embedded in a polymer film.

\section{Conclusions}

The in silico study of the modulation of emission properties of TPA-BMO requires the use and configuration of tailored computational chemistry methodologies based on molecular dynamics, (TD-)DFT and ONIOM 


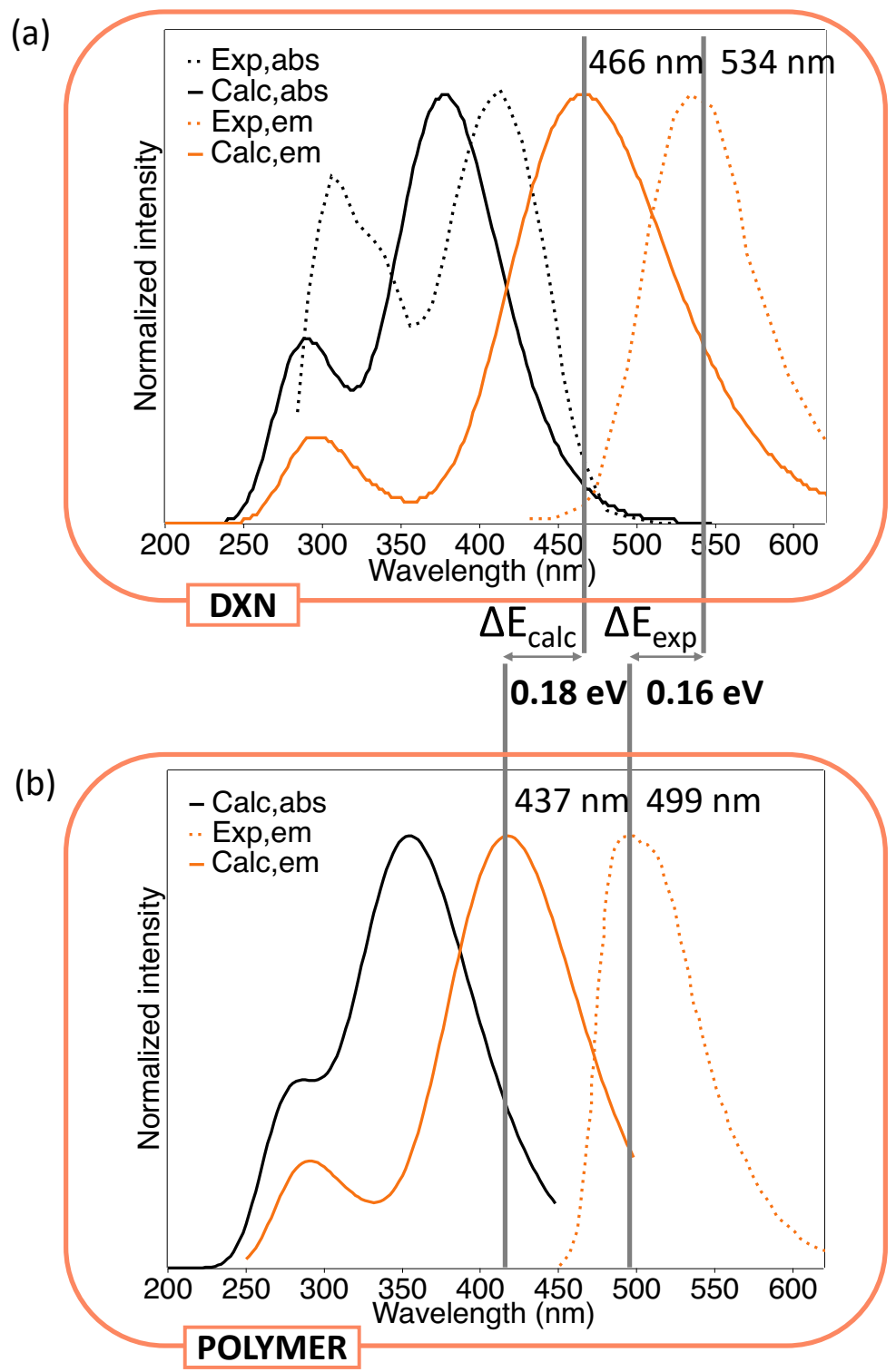

Figure 13: Comparison of optical properties of TPA-BMO in DXN (a) and in the polybutadiene polymer matrix (b)

QM/QM' calculations. As revealed by this work, a particular attention should be paid to the modeling of the environment. Indeed, for a fluorophore in solution, solvatochromic effects can be reproduced with the help of PCM implicit model. In this context, the moderate fluorescence quenching observed in solution with the increase of the solvent polarity is due to the modulation of the photophysical energy dissipation caused by low-frequency vibrational modes. For TPA-BMO molecules forming an aggregate, our study revealed the importance of analyzing and comparing the properties of the molecules located at the center and at the 
periphery of the structure. In this context, to understand the observed AIE phenomenon, the competition between the restriction of intramolecular vibration (RIV) mechanism and the moderate energy loss through excitonic couplings was investigated. For TPA-BMO, our study revealed that the hindrance of the vibrational modes involved in the non-radiative decay process in solution is the predominant effect. Finally, we used a recently set-up methodology which is able to mimic an in situ chemical polymerization and thus to propose a realistic arrangement of a chromophore embedded in the polymer film. Careful analysis of MD trajectories highlighted the impact of the polybutadiene matrix on the structural and optical properties through mechanical and electronic effects. Although there is a loss of planarity and flexibility of the fluorophore within the polymer, the vibrational modes mainly involved in the energy dissipation during the internal conversion process that were identified in solution are still present. This in agreement with the absence of fluorescence quenching experimentally observed for TPA-BMO embedded in a polybutadiene film. To conclude, a careful modeling of the fluorophore environment and a combination of theoretical strategies allow the investigation of the different photophysical processes at the origin of the modulation of the emission properties and provide a qualitative understanding of the experimental observations. The design of novel AIE functional materials can thus benefit from the multi-scale and multi-environment computational approach developed in this study.

\section{Conflicts of Interest}

There are no conflicts to declare.

\section{Supporting Information}

See Supporting Information for the (1) benchmark of the exchange-correlation functional, (2) definition of charge transfer parameters, (3) validation of the GAFF force field, (4) set up of the computational scheme for the calculation of the optical properties of TPA-BMO within the aggregate and the polymer matrix, (5) additional information for the properties of TPA-BMO in solution, aggregate and polymer.

\section{Acknowledgment}

This work was granted access to the HPC resources of CINES and IDRIS under the allocations 2019 and 2020-A0010810135 made by GENCI (Grand Equipement National de Calcul Intensif).

\section{Keywords}

Aggregation Induced Emission ; Computational Chemistry 


\section{References}

[1] R. Epple and T. Förster. Untersuchungen über Löschung und Polarisationsgrad der Fluoreszenz in Lösung. Zeitschrift für Elektrochemie, Berichte der Bunsengesellschaft für physikalische Chemie, 58(9):783-787, 1954.

[2] T. Förster and K. Kasper. Ein Konzentrationsumschlag der Fluoreszenz des Pyrens. Zeitschrift für Elektrochemie, Berichte der Bunsengesellschaft für physikalische Chemie, 59(10):976-980, 1955.

[3] J. Luo, Z. Xie, J. W. Y. Lam, L. Cheng, H. Chen, C. Qiu, H. S. Kwok, X. Zhan, Y. Liu, D. Zhu, and B. Z. Tang. Aggregation-Induced Emission of 1-methyl-1,2,3,4,5-pentaphenylsilole. Chem. Commun., pages 1740-1741, 2001.

[4] Z. Yang, W. Qin, N. L. C. Leung, M. Arseneault, J. W. Y. Lam, G. Liang, H. H. Y. Sung, I. D. Williams, and B. Z. Tang. A mechanistic study of AIE processes of TPE luminogens: intramolecular rotation vs. configurational isomerization. J. Mater. Chem. C, 4:99-107, 2016.

[5] J. Mei, N.L.C. Leung, R.T.K. Kwok, J.W.Y. Lam, and B. Z. Tang. Aggregation-Induced Emission: Together we shine, united we soar! Chem. Rev., 115:11718-11940, 2015.

[6] Y. Chen, J. W. Y. Lam, R. T. K. Kwok, B. Liu, and B. Z. Tang. Aggregation-Induced Emission: fundamental understanding and future developments. Mater. Horiz., 6:428-433, 2019.

[7] R. Crespo-Otero, Q. Li, and L. Blancafort. Exploring potential energy surfaces for Aggregation-Induced Emission—from solution to crystal. Chem. Asian J., 14(6):700-714, 2019.

[8] Y. Zhang, M. Jiang, G.-C. Han, K. Zhao, B.Z. Tang, and K. S. Wong. Solvant effect and two-photon optical properties of triphenylamine-based Donor-Acceptor fluorophores. J. Phys. Chem. C, 119:27630$27638,2015$.

[9] M. Jiang, Z. He, Y. Zhang, H. H. Y. Sung, J. W. Y. Lam, Q. Peng, Y. Yan, K. S. Wong, I. D. Williams, Y. Zhao, and B. Z. Tang. Development of benzylidene-methyloxazolone based AIEgens and decipherment of their working mechanism. J. Mater. Chem. C, 5:7191-7199, 2017.

[10] Y. Yilin Zhang, M. Jiang, T. Han, X. Xiao, W. Chen, L. Wang, K.S. Wong, R. Wang, B.Z. Wang, K. Tang, and K. Wu. Aggregation-Induced Emission luminogens as color converters for visible-light communication. ACS. Appl. Mater. Interfaces, 10:34418-34426, 2018.

[11] T. Han, C. Gui, J.W.Y . Lam, M. Jiang, N. Xie, R.T.K . Kwok, and B. Z. Tang. High-contrast visualization and differentiation of microphase separation in polymer blends by fluorescent aie probes. Macromolecules, 50:5807-5815, 2017. 
[12] R. Mori, G. Iasilli, M. Lessi, A. B. Muñoz-García, M. Pavone, F. Bellina, and A. Pucci. Luminescent solar concentrators based on PMMA films obtained from a red-emitting ATRP initiator. Polym. Chem., 9:1168-1177, 2018.

[13] G. Iasilli, A. Battisti, F. Tantussi, F. Fuso, M. Allegrini, G. Ruggeri, and A. Pucci. Aggregation-Induced Emission of tetraphenylethylene in styrene-based polymers. Macromol. Chem. Phys., 215(6):499-506, 2014.

[14] L. Le Bras, C. Adamo, and A. Perrier. Modeling the modulation of emission behavior in E/Z isomers of dipyrrolyldiphenylethene : from molecules to nanoaggregates. J. Phys. Chem. C, 121:25603-25616, 2017.

[15] L. Le Bras, K. Chaitou, S. Aloïse, C. Adamo, and A. Perrier. Aggregation-Caused Quenching versus Cristallization Induced Emission in thiazolo[5,4-b]thieno[3,2-e]-pyridine (TTP) derivatives : theoretical insight. Phys. Chem. Chem. Phys, 21(10):46-56, 2019.

[16] L. Le Bras, C. Adamo, and A. Perrier. In silico investigation of the aggregation-caused quenching: the "tolane-based molecule" case. ChemPhotoChem, 3:1-11, 2019.

[17] D. Presti, L. Wilbraham, C. Targa, F. Labat, A. Pedone, M. C. Menziani, I. Ciofini, and C. Adamo. Understanding aggregation-induced emission in molecular crystals: Insights from theory. J. Phys. Chem. C, 121(10):5747-5752, 2017.

[18] L. Wilbraham, M. Louis, D. Alberga, A. Brosseau, R. Guillot, F. Ito, F. Labat, R. Métivier, C. Allain, and I. Ciofini. Revealing the origins of mechanically induced fluorescence changes in organic molecular crystals. Adv. Mater., 30(28):1800817, 2018.

[19] L. Le Bras, C. Lemarchand, S. Aloïsse, C. Adamo, N. Pineau, and A. Perrier. Modeling photonastic materials: A first computational study. J. Chem. Theory Comput, 16(11):7017-7032, 2020.

[20] M. J. Frisch, G. W. Trucks, H. B. Schlegel, G. E. Scuseria, M. A. Robb, J. R. Cheeseman, G. Scalmani, V. Barone, G. A. Petersson, H. Nakatsuji, X. Li, M. Caricato, A. V. Marenich, J. Bloino, B. G. Janesko, R. Gomperts, B. Mennucci, H. P. Hratchian, J. V. Ortiz, A. F. Izmaylov, J. L. Sonnenberg, D. WilliamsYoung, F. Ding, F. Lipparini, F. Egidi, J. Goings, B. Peng, A. Petrone, T. Henderson, D. Ranasinghe, V. G. Zakrzewski, J. Gao, N. Rega, G. Zheng, W. Liang, M. Hada, M. Ehara, K. Toyota, R. Fukuda, J. Hasegawa, M. Ishida, T. Nakajima, Y. Honda, O. Kitao, H. Nakai, T. Vreven, K. Throssell, J. A. Montgomery, Jr., J. E. Peralta, F. Ogliaro, M. J. Bearpark, J. J. Heyd, E. N. Brothers, K. N. Kudin, V. N. Staroverov, T. A. Keith, R. Kobayashi, J. Normand, K. Raghavachari, A. P. Rendell, J. C. Burant, S. S. Iyengar, J. Tomasi, M. Cossi, J. M. Millam, M. Klene, C. Adamo, R. Cammi, J. W. Ochterski, R. L. 
Martin, K. Morokuma, O. Farkas, J. B. Foresman, and D. J. Fox. Gaussian16 Revision A.03. 2016. Gaussian Inc. Wallingford CT.

[21] J.P. Perdew, M. Ernzerhof, and K. Burke. Rationale for mixing exact exchange with density functional approximations. J. Chem. Phys., 105(22):9982-9985, 1996.

[22] C. Adamo and V. Barone. Toward reliable density functional methods without adjustable parameters: The PBE0 model. J. Chem. Phys., 110(13):6158-6170, 1999.

[23] C. Lee, W. Yang, and R.G. Parr. Development of the Colle-Salvetti correlation-energy formula into a functional of the electron density. Phys. Rev. B, 37:785-789, Jan 1988.

[24] T. Yanai, D.P. Tew, and N.C. Handy. A new hybrid exchange correlation functional using the coulombattenuating method (CAMB3LYP). Chem. Phys. Lett., 2004:51-57, 393.

[25] Y. Zhao and D.G. Truhlar. A new local density functional for main-group thermochemistry, transition metal bonding, thermochemical kinetics, and noncovalent interactions. J. Chem. Phys., 125(19):194101, 2006.

[26] J. Tomasi, B. Mennucci, and R. Cammi. Quantum mechanical continuum solvation models. Chem. Rev., 105(8):2999-3094, 2005.

[27] D. Jacquemin, T. Le Bahers, C. Adamo, and I. Ciofini. What is the "best" atomic charge model to describe through-space charge-transfer excitations? Phys. Chem. Chem. Phys., 14:5383-5388, 2012.

[28] T. Le Bahers, C. Adamo, and I. Ciofini. A qualitative index of spatial extent in charge-transfer excitations. J. Chem. Theory Comput., 7(8):2498-2506, 2011.

[29] Y.-C. Duan, Y. Wu, J.-L. Jin, D.-M. Gu, Y. Geng, M. Zhang, and Z.-M. Su. Influence of aggregation on the structure and fluorescent properties of a tetraphenylethylene derivative: a theoretical study. ChemPhysChem, 18(7):755-762, 2017.

[30] D.A. Case, D.S. Cerutti, T.E. III Cheatham, T.A. Darden, R.E. Duke, T.J. Giese, H. Gohlke, A.W. Goetz, D. Greene, N. Homeyer, S. Izadi, A. Kovalenko, T.S. Lee, S. LeGrand, P. Li, C. Lin, J. Liu, T. Luchko, R. Luo, D. Mermelstein, K.M. Merz, G. Monard, H. Nguyen, I. Omelyan, A. Onufriev, F. Pan, R. Qi, D.R. Roe, A. Roitberg, C. Sagui, C.L. Simmerling, W.M. Botello-Smith, J. Swails, R.C. Walker, J. Wang, R.M. Wolf, X. Wu, L. Xiao, D.M. York, and P.A. Kollman. Amber18. University of California : San Francisco CA, 2018.

[31] J. Wang, R. M. Wolf, J. W. Caldwell, P. A. Kollman, and D. A. Case. Development and testing of a general AMBER force field. J. Comput. Chem., 25:1157-1174, 2004.

[32] W. L. Jorgensen, J. Chandrasekhar, J. D. Madura, R. W. Impey, and M. L. Klein. Comparison of simple potential functions for simulating liquid water. J. Chem. Phys., 79(2):926-935, 1983. 
[33] U. Essmann, L. Perera, M. L. Berkowitz, T. Darden, H. Lee, and L. G. Pedersen. A smooth particle mesh Ewald method. J. Chem. Phys., 103:8577-8593, 1995.

[34] T. Darden, D. York, and L. Pedersen. Particle mesh Ewald: An N.log(N) method for Ewald sums in large systems. J. Chem. Phys., 98(12):10089-10092, 1993.

[35] H. J. C. Berendsen, J. P. M. Postma, W. F. van Gunsteren, A. DiNola, and J. R. Haak. Molecular dynamics with coupling to an external bath. J. Chem. Phys., 81(8):3684-3690, 1984.

[36] D. R. Roe and T. E. Cheatham. PTRAJ and CPPTRAJ: Software for Processing and Analysis of Molecular Dynamics Trajectory Data. J. Chem. Theory Comput., 9(7):3084-3095, 2013.

[37] L. Martínez, R. Andrade, E. G. Birgin, and J. M. Martínez. Packmol: A package for building initial configurations for molecular dynamics simulations. J. Comput. Chem., 30(13):2157-2164, 2009.

[38] X. Zheng, Q. Peng, L. Zhu, Y. Xie, X. Huang, and Z. Shuai. Unraveling the aggregation effect on amorphous phase AIE luminogens: a computational study. Nanoscale, 8:15173-15180, 2016.

[39] H. P. Hratchian, A. V. Krukau, P. V. Parandekar, M. J. Frisch, and K. Raghavachari. QM:QM embedding using electronic densities within an ONIOM framework: Energies and analytic gradients. J. Chem. Phys., 135(1):014105, 2011.

[40] T. Vreven, B. Mennucci, C. O. da Silva, K. Morokuma, and J. Tomasi. The ONIOM-PCM method: Combining the hybrid molecular orbital method and the polarizable continuum model for solvation. application to the geometry and properties of a merocyanine in solution. J. Chem. Phys., 115(1):62-72, 2001.

[41] S. J. Mo, T. Vreven, B. Mennucci, K. Morokuma, and J. Tomasi. Theoretical study of the $\mathrm{sn}_{2}$ reaction of $\mathrm{cl}-\left(\mathrm{h}_{2} \mathrm{O}\right)+\mathrm{ch}_{3} \mathrm{cl}$ using our own n-layered integrated molecular orbital and molecular mechanics polarizable continuum model method (ONIOM, PCM). Theor. Chem. Acc., 111:154-161, 2004.

[42] N. Pineau, L. Soulard, L. Colombet, T. Carrard, A. Pelle, Ph. Gillet, and J. Clerouin. Molecular dynamics simulations of shock compressed heterogeneous materials. II. the graphite/diamond transition case for astrophysics applications. J. Appl. Phys., 117:115902, 2015.

[43] J. H. Los, N. Pineau, G. Chevrot, G. Vignoles, and J.-M. Leyssale. Formation of multiwall fullerenes from nanodiamonds studied by atomistic simulations. Phys. Rev. B, 80:155420, 2009.

[44] T. Dreher, N. Pineau, E. Bourasseau, P. Malfreyt, L. Soulard, and C. A. Lemarchand. Anisotropic surface stresses of a solid/fluid interface: Molecular dynamics calculations for the copper/methane interface. $J$. Chem. Phys., 151:244703, 2019.

[45] C. A. Lemarchand, D. Bousquet, B. Schnell, and N. Pineau. A parallel algorithm to produce long polymer chains in molecular dynamics. J. Chem. Phys., 150(22):224902, 2019. 
[46] W. L. Jorgensen, D. S. Maxwell, and J. Tirado-Rives. Development and testing of the OPLS allatom force field on conformational energetics and properties of organic liquids. J. Am. Chem. Soc., 118:11225-11236, 1996.

[47] C. F. Chen. Dielectric Properties of Polybutadiene and its Reinforced Composites at Room and Elevated Temperature. Electrical Insulation Conference, EIC 10th, 1971.

[48] Z. Pu. Polymer Data Handbook, trans-1,4-polybutadiene. Oxford University Press, Oxford, 1999.

[49] M. Pegoraro and K. Mitoraj. Dielectric properties of trans-1,4-polybutadiene. Makromol. Chem., 61:132, 1963.

[50] W Bartkowiak. Solvatochromism and Nonlinear Optical Properties of Donor-acceptor $\pi$ Conjugated Molecules, volume 1, pages 299-318. Springer: Dordrecht, The Netherlands, 2006.

[51] L. Lin, J. Fan, L. Cai, and C.-K. Wang. Theoretical perspective of the excited state intramolecular proton transfer for a compound with aggregation induced emission in the solid phase. RSC Adv., 7:44089-44096, 2017.

[52] Z. Shuai. Thermal vibration correlation function formalism for molecular excited state decay rates. Chin. J. Chem, 38(11):1223-1232, 2020.

[53] C. Curutchet and B. Mennucci. Toward a molecular scale interpretation of excitation energy transfer in solvated bichromophoric systems. J. Am. Chem. Soc, 47:16733-16744, 2005.

[54] C. Curutchet, G. D. Scholes, B. Mennucci, and R. Cammi. How solvent controls electronic energy transfer and light harvesting: Toward a quantum mechanical description of reaction field and screening effects. J. Phys. Chem. B, 111(46):13253-13265, 2007. 